\title{
MORPHOLOGY AND CONFORMATION OF POLYTHIOPHENE DERIVATIVES IN ANISOTROPIC CORE-SHELL NANOCOMPOSITES AND SOLUTION
}

\author{
A Thesis \\ presented to \\ the Faculty of California Polytechnic University, \\ San Luis Obispo \\ In Partial Fulfillment \\ of the Requirements for the Degree \\ Master of Science in Polymers and Coatings Sciences \\ by \\ Neil Redeker \\ November 2013
}


(C) 2014

Neil David Redeker

ALL RIGHTS RESERVED 
COMMITTEE MEMBERSHIP

TITLE:

Morphology and Conformation of

Polythiophene Derivatives in Anisotropic CoreShell Nanocomposites and Solution

AUTHOR:

Neil Redeker

DATE SUBMITTED: November 2013

CHAIR:

Shanju Zhang, PhD

Assistant Professor of Chemistry

MEMBER:

Raymond Fernando, PhD

Professor of Chemistry, Director of Polymers

and Coatings Program

MEMBER:

John Hagen, PhD

Professor of Chemistry 


\begin{abstract}
Morphology and Conformation of Polythiophene Derivatives in Anisotropic Core-Shell Nanocomposites and Solution
\end{abstract}

\title{
Neil Redeker
}

Conjugated semiconducting polymers have garnered substantial interest in recent years due to the potential for use in various applications, particularly in the field of electronic devices such as photovoltaic cells and light emitting diodes. Conjugated polymers offer numerous advantages in these applications, including low cost and high flexibility, but electronic devices based on these materials are currently limited by poor performance. Because of these limitations, increased focus has been placed on improving conjugated polymers for use in commercially viable products. Here, a novel core shell hybrid nanocomposite based on anisotropic zinc oxide nanowires and a side-chain functionalized polythiophene is reported. This nanocomposite exhibits confirmed covalent side-on linkage between the polymer and the nanowires, and the crystalline, thermal and photophysical properties of the nanocomposite are investigated, revealing elongated conjugation length in the polymer backbone, increased crystallinity and thermal stability and rapid charge transfer. Additionally, the conformational transitions of side-chain 
functionalized polythiophenes are investigated in dilute solution through the use of ultraviolet-visible absorption spectrophotometry. A coil-to-rod conformational transition is identified, and is found to be induceable through temperature and solvent changes. Study into the kinetics of the transition reveals a first-order rate law, and the effects of polymer structure on the conformational transition are substantiated. 


\section{ACKNOWLEDGMENTS}

Firstly I would like to thank Dr. Shanju Zhang for being such an excellent advisor to me and the rest of our research group. His constant effort to expand our academic and research experience as well as his invaluable guidance and support are primarily what have made this work possible, and I am extremely thankful for his advice, direction and determination to see us succeed both in the lab and out of it. Without Dr. Zhang I would not be here today.

I would like to give special thanks to Dr. Ray Fernando for introducing me to polymers and the PCS master's program. His continued support and direction for all of the PCS masters students is a huge part of what makes this program such a great experience.

I would also like to thank Dr. John Hagen, who was my first chemistry professor at Cal Poly. I was unsure of my major when I applied, but the passion that Dr. Hagen showed in his general chemistry lectures inspired me to continue on as a biochemistry major.

I would like to thank my colleagues Cameron Danesh and Taylor Wagner as well as the rest of the members of Dr. Zhang's research group for their help and insight. 
Additionally I would like to thank the Polymers and Coatings program as well as the Chemistry and Biochemistry department for the support throughout my time here at Cal Poly.

Finally, this project was made possible by funding from the Bill Moore Coatings Research Fellowship Award and the National Science Foundation. 


\section{TABLE OF CONTENTS}

Page

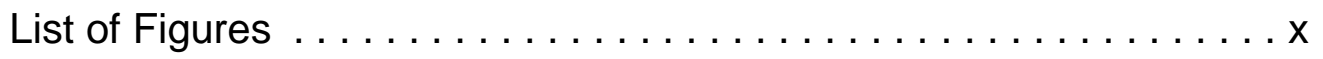

1. Introduction. ......................

1.1. History of the Solar Cell. . . . . . . . . . . . . 1

1.2. Polymer Solar Cells. . . . . . . . . . . . . . . 3

1.3 Improving Operational Lifetimes of Polymer Photovoltaic Devices ........................11

1.4 Improving Efficiencies of Polymer Photovoltaic Devices . . 16

1.5 Controlling Polymer Alignment and Crystallinity. . . . . . . 20

1.6 Hybrid Solar Cells. . . . . . . . . . . . . . . . . . 24

1.7 Hybrid Core-Shell Nanocomposites . . . . . . . . . . . . . 27

$1.8 \quad$ Research Plan . . . . . . . . . . . . . . . . . 30

2. Methods and Materials . . . . . . . . . . . . . . . . . 32

2.1. Material Preparation . . . . . . . . . . . . . . . . 32

2.1.1. Preparation of Zinc Oxide Nanowires (ZnO-NW) . . 32

2.1.2. Preparation of Nanocomposites . . . . . . . . 33

2.1.3. Preparation of Pristine Polymer Samples . . . . . . 35

2.2. Characterization and Analysis . . . . . . . . . 36

2.2.1 UV-Visible Absorption Spectrophotometry . . . . . 36 
2.2.2 UV-Visible Emission Spectrophotometry..... . . 37

2.2.3 Fourier Transform Infrared Spectroscopy . . . . . . . 38

2.2.4 Thermogravimetric Analysis $\ldots \ldots \ldots \ldots \ldots 38$

2.2.5 Transmission Electron Microscopy . . . . . . . . 39

2.2.6 X-Ray Diffraction . . . . . . . . . . . . . 39

3. Results and Discussion $\ldots \ldots \ldots \ldots \ldots \ldots \ldots \ldots \ldots \ldots \ldots$

3.1. Nanocomposite Characterization . . . . . . . . . 41

3.1.1. Confirmation of Side-On Chemical Core-Shell

Linkage.................... 41

3.1.2. Nanocomposite Thermal Characterization ...... 44

3.1.3. Nanocomposite Shell: Spectral Characterization . . 46

3.2. Conformational Characterization of P3CATs . . . . . . 55

3.2.1 Dilute Solution Spectrophotometry of P3CPenT

Polymer .................... 55

3.2.2 Effect of Side-Chain Length on Conformation .... . 61

3.2.3 Kinetics of Conformational Transition in P3CPenT . 64

3.3. Research Outlook. .................. 68

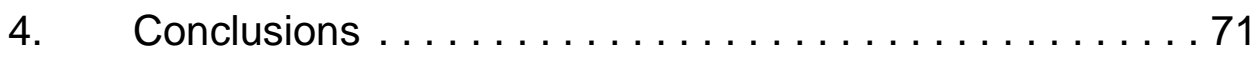

5. References..................... 73 


\section{LIST OF FIGURES}

Figure Page

1.1 The band gap of a material determines its conductivity.

The band gap may be thought of as the minimum

amount of energy required to excite an electron from the

valence band (red) to the conduction band (blue) ...... 4

1.2 There are many different types of CPs. Shown here are

three of the most commonly used CPs in polymer based

solar cells: polyacetylene (a), polyphenylvinylene (b)

and polythiophene (c). Although these polymers vary

extensively in their structure, all are somewhat

conductive as a result of their conjugated backbones . . . . 6

1.3 The photovoltaic effect is the phenomenon responsible

for the generating current in solar cells. It involves the

generation of excitons by incident light, which can then

either decay (a) or diffuse to the donor/accepter

interfaceof the $p-n$ junction. Following this, local fields

split the exciton into its constituent charge carriers, which

are then free to propagate through the device until they

reach their respective electrodes $\ldots \ldots \ldots \ldots \ldots \ldots$ 
1.4 Efficiency records for various photovoltaic cells produced by research groups, organized by material type. . . . . 12

1.5 Photoinitiated oxidation is a major concern for all polymer

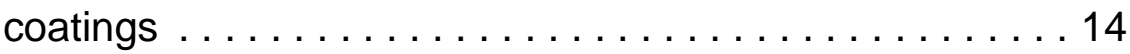

1.6 A bulk heterojunction device model (b) allows for increased interfacial area but results in nonideal charge pathways. The nanoscale periodicity of the ordered bulk heterojunction model (a) solves this issue while maintaining high interfacial area $\ldots \ldots \ldots \ldots \ldots 17$

1.7 Solvent interactions influence the conformation of the polymer. Shown here, P3HT adopts the more rigid rod conformation in poor solvents (right) and a relaxed coil conformation in good solvents (left) . . . . . . . . . 21

1.8 The surface area to volume ratio for a given particle increases exponentially as particle size decreases . . . . 26

1.9 Core-shell nanocomposites consist of a nanomaterial core attached to a surrounding polymer shell $\ldots \ldots \ldots 28$

1.10 End-on polymer shell attachment results in hairpin folding of the polymer backbone, producing a torturous charge carrier pathway through the polymer shell . . . . . 29 
2.1 The side-on chemical grafting of the nanocomposite is made possible by the reaction of the carboxylic acid functional side-groups with the $\mathrm{ZnO}-\mathrm{NWs} \ldots \ldots \ldots . .34$

3.1 $\quad$ FT-IR spectra of pristine polymer films cast from pyridine (a) and DMSO (b) and dry bulk nanocomposite produced from solutions in pyridine (c) and DMSO (d) $\ldots \ldots \ldots \ldots \ldots \ldots \ldots \ldots \ldots \ldots \ldots$

3.2 Low resolution (a) and high resolution (b, c) TEM images of hybrid nanocomposite samples $\ldots \ldots \ldots \ldots \ldots \ldots 43$

3.3 TGA heating curves for nanocomposite samples prepared in DMSO using P3CPenT:ZnO initial concentrations of 1:9 (a), 3:7 (b) and 1:1 (c), with bulk pristine P3CPenT polymer $(\mathrm{d})$ as a reference ....... 44

3.4 Normalized TGA heating curves for bulk pristine P3CPenT (a, red) and bulk 1:1 nanocomposite prepared in DMSO $(b$, black $) \ldots \ldots \ldots \ldots \ldots \ldots 46$ 
3.5 Normalized UV-vis absorption spectra of P3CPenT

polymer (a), nanocomposite prepared in DMSO (b) and

$\mathrm{ZnO}$ nanowires (c). All spectra shown collected from

DMSO solutions $(10 \mathrm{ug} / \mathrm{mL}) \ldots \ldots \ldots \ldots \ldots \ldots 4$

3.6 Normalized UV-Vis absorbance of nanocomposite prepared in DMSO (a, red) and pyridine (b, black) (10 $\mathrm{ug} / \mathrm{mL}) \ldots \ldots \ldots \ldots \ldots \ldots \ldots \ldots \ldots \ldots \ldots$

3.7 Normalized UV-Vis absorbance of P3CPenT polymer in DMSO (a, red) and pyridine (b, black) $(10 \mathrm{ug} / \mathrm{mL}) \ldots \ldots 48$

3.8 XRD patterns of $\mathrm{ZnO}$ nanowires (a), DMSO prepared nanocomposite (b), Pyridine prepared nanocomposite (c) and bulk dry P3CPenT polymer (d) . . . . . . . 50

3.9 Normalized XRD patterns for spin cast P3CPenT films produced from polymer solutions in pyridine (a) and DMSO (b) $\ldots \ldots \ldots \ldots \ldots \ldots \ldots \ldots \ldots \ldots \ldots \ldots \ldots \ldots \ldots \ldots \ldots$ 
3.10 UV-visible photoluminescence spectra for pristine P3CPenT polymer (a, red), physical mixture of $\mathrm{ZnO}-\mathrm{NW}$ and pristine $\mathrm{P} 3 \mathrm{CPenT}$ polymer (b, blue), nanocomposite prepared in pyridine (c, orange) and nanocomposite prepared in DMSO (d, black). All samples measured in DMSO (12.5 ug/mL polymer). Inset displays normalized emission intensity for all samples to highlight differences in peak positions. . . . . . . . . . . . . 53

3.11 UV-Visible absorption spectra of pristine P3CPenT dissolved in solutions with varying DMSO:EtOH solvent ratios highlighting the solvatochromism of $\mathrm{P} 3 \mathrm{CPent}$. All spectra collected at $20^{\circ} \mathrm{C}(25 \mathrm{ug} / \mathrm{mL}) \ldots \ldots \ldots \ldots \ldots 57$

3.12 UV-Visible absorption spectra of pristine P3CPenT dissolved in solutions with varying DMSO:EtOH solvent ratios highlighting the solvatochromism of $\mathrm{P} 3 \mathrm{CPent}$. All spectra collected at $80^{\circ} \mathrm{C}(25 \mathrm{ug} / \mathrm{mL}) \ldots \ldots \ldots \ldots \ldots 58$

3.13 UV-Visible absorption spectra of pristine P3CPenT polymer in a solution of $80 \%$ DMSO:20\% EtOH at varying temperatures $(25 \mathrm{ug} / \mathrm{mL}) \ldots \ldots \ldots \ldots \ldots 59$ 
3.14 UV-Visible absorption heating curve (a) and cooling curve (b) for P3CPenT in 80\% DMSO: 20\% EtOH (25

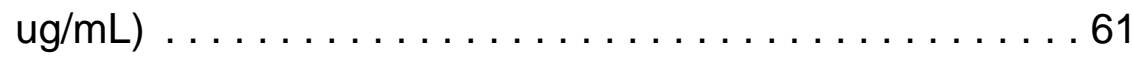

3.15 UV-visible absorption spectra of P3CAT polymers at $20 \mathrm{C}$ in $100 \%$ DMSO:0\% EtOH solution $(25 \mathrm{ug} / \mathrm{mL}) \ldots \ldots 62$

3.16 UV-Visible absorption spectra of P3CHexylT (A), P3CPenT (B), P3CButylT (C) and P3CPropylT (D) in solutions of varying DMSO:EtOH solvent ratios at $20^{\circ} \mathrm{C}$ $(25 \mathrm{ug} / \mathrm{mL})$ 63

3.17 Change in absorbance $(\lambda=590)$ with respect to time for 40 ug/mL P3CPenT (a), 25 ug/mL P3CPenT (b), 15 ug/mL P3CPenT (c), $10 \mathrm{ug} / \mathrm{mL}$ P3CPenT (d) and 5 ug/mL P3CPenT (e) . . . . . . . . . . . . . 64

3.18 Initial rate plot fitted with a power function (black line) determined from the kinetic data for P3CPenT shown in Figure $13.17 \ldots \ldots \ldots \ldots \ldots \ldots \ldots \ldots \ldots 65$

3.19 Time dependent chromism of new P3CPenT samples in 100\% DMSO:0\% EtOH solution $(25 \mathrm{ug} / \mathrm{mL}) \ldots \ldots \ldots 66$ 
3.20 Visual picture of ZnO-NW (left), P3CPenT

nanocomposite (center) and P3CProT nanocomposite

(right) $\ldots \ldots \ldots \ldots \ldots \ldots \ldots \ldots \ldots \ldots \ldots \ldots \ldots \ldots \ldots \ldots \ldots$

3.21 Optical imaging of the core-shell nanocomposites under cross polars (represented by the arrows) suggests the formation of a lyotropic liquid crystalline phase, as indicated by the strong birefringence $\ldots \ldots \ldots \ldots \ldots 70$ 


\section{Introduction}

\subsection{History of the Solar Cell}

Solar cells, also called photovoltaic cells, are an important potential source of energy. These photovoltaic devices can transform energy in sunlight into electrical current, essentially allowing us to directly harness the power of the sun. The potential for these types of devices is huge: the earth's surface receives about 89,000 TW of solar power ${ }^{1}$. To give an idea of just how much energy this is, the world's total energy consumption rate in 2001 was approximately 14 TW ${ }^{1}$. In other words, in order to provide for the entire energy usage of the human race currently, we would need to harness less than $0.1 \%$ of the solar energy available to us. Additionally, solar energy is a renewable source of energy; that is to say that it cannot be exhausted and is naturally occurring, allowing for permanent exploitation. Because of these compelling potential advantages, solar power has been a particularly popular subject of research in recent years.

The discovery of the solar cell dates back to the $19^{\text {th }}$ century: French physicist Edmond Becquerel is credited with construction of the first solar cell in 1839, after he discovered that two different brass plates immersed in liquid produced a current when light was applied ${ }^{2}$. Later, in the 1880's, C. E. Fritts was the first to construct a solid state solar cell, 
consisting of amorphous selenium on a metal backing, covered by a gold leaf film, remarking that the device produced a current "that is consistent, constant, and of considerable force-with exposure to sunlight" ${ }^{3}$. Unfortunately, these discoveries were met with considerable skepticism, as quantum mechanics had not yet been discovered and there were no theories to explain these phenomena. It was not until 1954 that the first practical solar cell was developed, this time made of crystalline silicon and produced at Bell Laboratories ${ }^{4}$.

At first, solar cells were used mainly as power sources for satellites, because despite their high power-to-weight ratios, they were very expensive compared to other energy sources. However, improvements in device design and production, as well as the development of new types of solar cells, have led to increased device power generation and decreased cost, with device cost per watt dropping from nearly $\$ 75 /$ watt at the start of commercial production in the 1970 's to under $1 \$ /$ watt today ${ }^{5}$. Several different types of solar cells exist, including the more traditional crystalline silicon based cells, as well as thin film cells based on amorphous silicon, $\mathrm{CdTe}, \mathrm{Cu}(\mathrm{In}, \mathrm{Ga}) \mathrm{Se}_{2}$ and even organic small molecules and polymers. In particular, organic semiconducting conjugated polymer (CP) based solar cells are a relatively recent invention which have generated significant 
interest and have the potential to solve many problems associated with solar power.

\subsection{Polymer Solar Cells}

Polymer solar cells are based on organic semiconducting polymers, long chain macromolecules containing chemically bonded, repeating molecular units with semiconducting electronic properties. The conductivity of CPs arises from the conjugated pi-bonds along the polymer backbone. These conjugated pi-bonds increase the overlap of $p$-orbitals along the backbone, increasing the mobility of electrons along the backbone by reducing the band gap of the material. The band gap of a material refers to the energy it takes to excite an electron in the material from the valence band, or the Highest Occupied Molecular Orbital (HOMO) to the conduction band, or the Lowest Unoccupied Molecular Orbital (LUMO), and may be thought of as a measure of the conductivity of the material (Figure 1.1). Insulators have a very large band gap which makes it very difficult for the electrons to be promoted into the conduction state, essentially binding them to their respective atoms, while semiconductors have a smaller band gap, requiring the electrons to be excited before they may be conducted. Metallic conductors have no band gap: the electrons essentially exist free of their respective orbitals and, in 
the presence of an electric potential, are free to travel down the potential, creating current. Although CPs are traditionally considered semiconducting materials, their conductivity can vary extensively based on a number of factors including the identity of the polymer repeat unit,

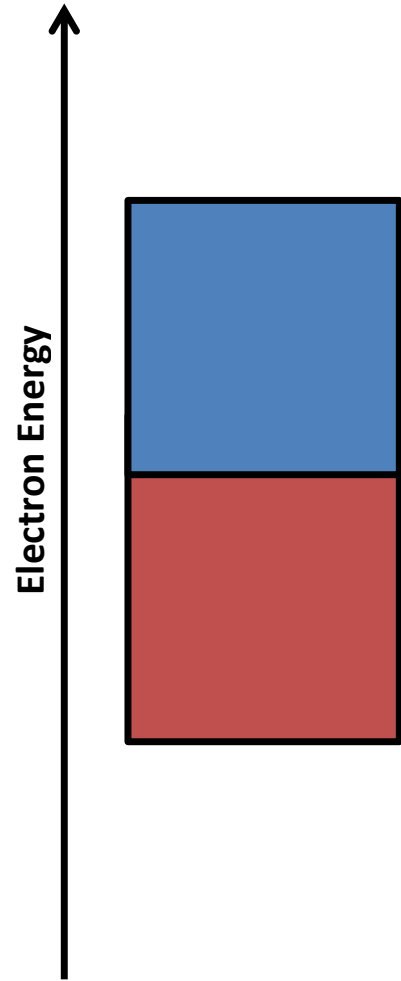

Conductor
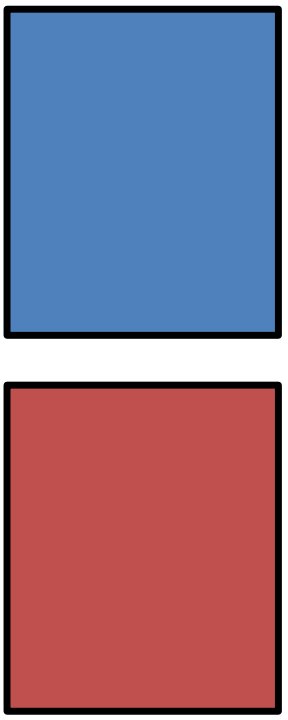

Semiconductor

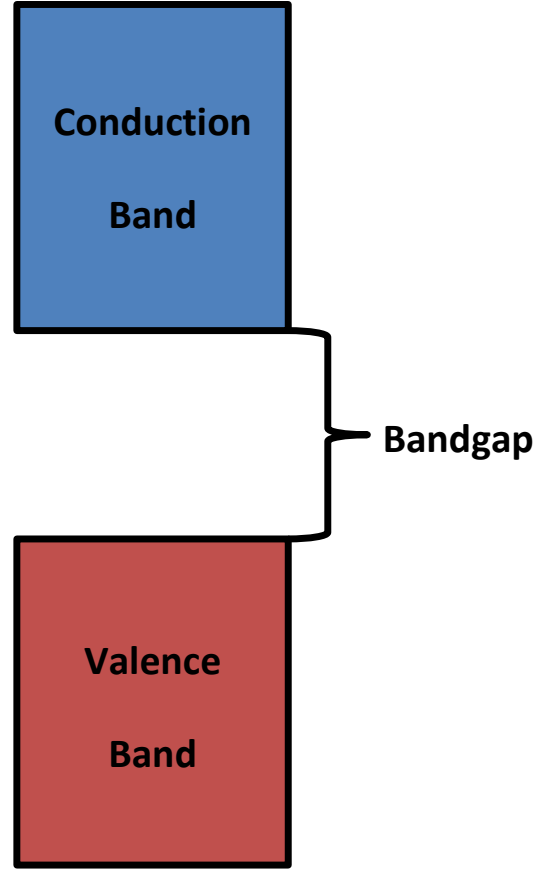

Insulator

Figure 1.1: The band gap of a material determines its conductivity. The band gap may be thought of as the minimum amount of energy required to excite an electron from the valence band (red) to the conduction band (blue). 
presence of doping agents, polymer conformation and crystalline orientation.

There are many different categories of CPs, including polyacetylenes, polyphenylenes, and polythiophenes (Figures 1.2a, b and c respectively). Polyacetylene was one of the first semiconducting polymers which was widely studied, and has a very high conductivity ${ }^{6}$. However, it is unstable under atmospheric conditions, oxidizing relatively readily in the presence of oxygen. Polyphenylenes also have excellent conductive properties, but unmodified polyphenylenes are relatively insoluble in organic solvents, making them difficult to be incorporated into electronic devices. Polyphenylvinylene (PPV), a modified polyphenylene with vinyl (double bond) bridges inserted between the phenyl rings, was adopted to solve this problem ${ }^{7}$. PPV and PPV derivatives exhibit good solubility in a variety of organic solvents, but unfortunately these molecules have relatively poor conductivity, and are also vulnerable to atmospheric oxidation. Because of these issues, polythiophenes have become the most commonly used CPs in polymer solar cells. 


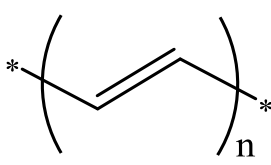

a) Polyacetylene

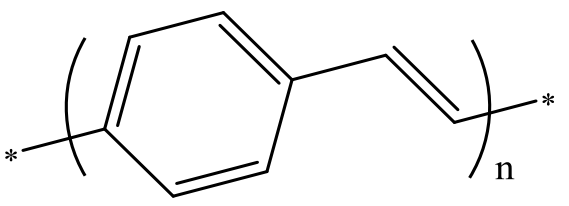

b) Polyphenylvinylene

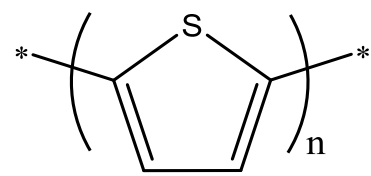

c) Polythiophene

Figure 1.2: There are many different types of CPs. Shown here are three of the most commonly used CPs in polymer based solar cells: polyacetylene (a), polyphenylvinylene (b) and polythiophene (c). Although these polymers vary extensively in their structure, all are somewhat conductive as a result of their conjugated backbones.

Similarly to polyphenylene, unsubstituted polythiophene is very difficult to solvate, but the solubility can be increased through the addition of functional solubilizing moieties, often alkyl side chains. These polyalkylthiophenes are relatively stable and have excellent optical and electrical properties. Poly(3-hexylthiophene), an hexylated polythiophene, is the most commonly used polythiophene in solar applications and represents the 'state of the art' in the field of polymer solar cells ${ }^{8}$.

As is the case with any solar cell, CP based solar cells generate current through a phenomenon known as the photovoltaic effect, which can be summed up in three steps: exciton generation, exciton splitting and charge carrier propagation (Figure 1.3). In the first step, incident photons 
with energy corresponding to the band gap of the material excite an electron from the valence band into the conduction band. The promotion of the valence electron into the conduction band also generates an electron hole, which may be thought of as an abstraction of the space that the electron occupied. The electron hole has a relative positive charge compared to that of the excited electron, which results in a weak coulombic attraction between the two charge carriers, forming a quasineutral charge carrier pair is known as an exciton. At this point, the exciton will either decay via thermal relaxation or photon re-emission unless the exciton dissociates, separating the electron and electron hole. In order for current to be generated in photovoltaic devices, the exciton must dissociate into its constituent charge carriers. This can be accomplished through the application of an electric field: essentially, the field causes the electron and the electron hole to be pulled in opposite directions as a result of their opposing charges. If the field is strong enough, it will result in the dissociation of the exciton into the electron and the electron hole. 


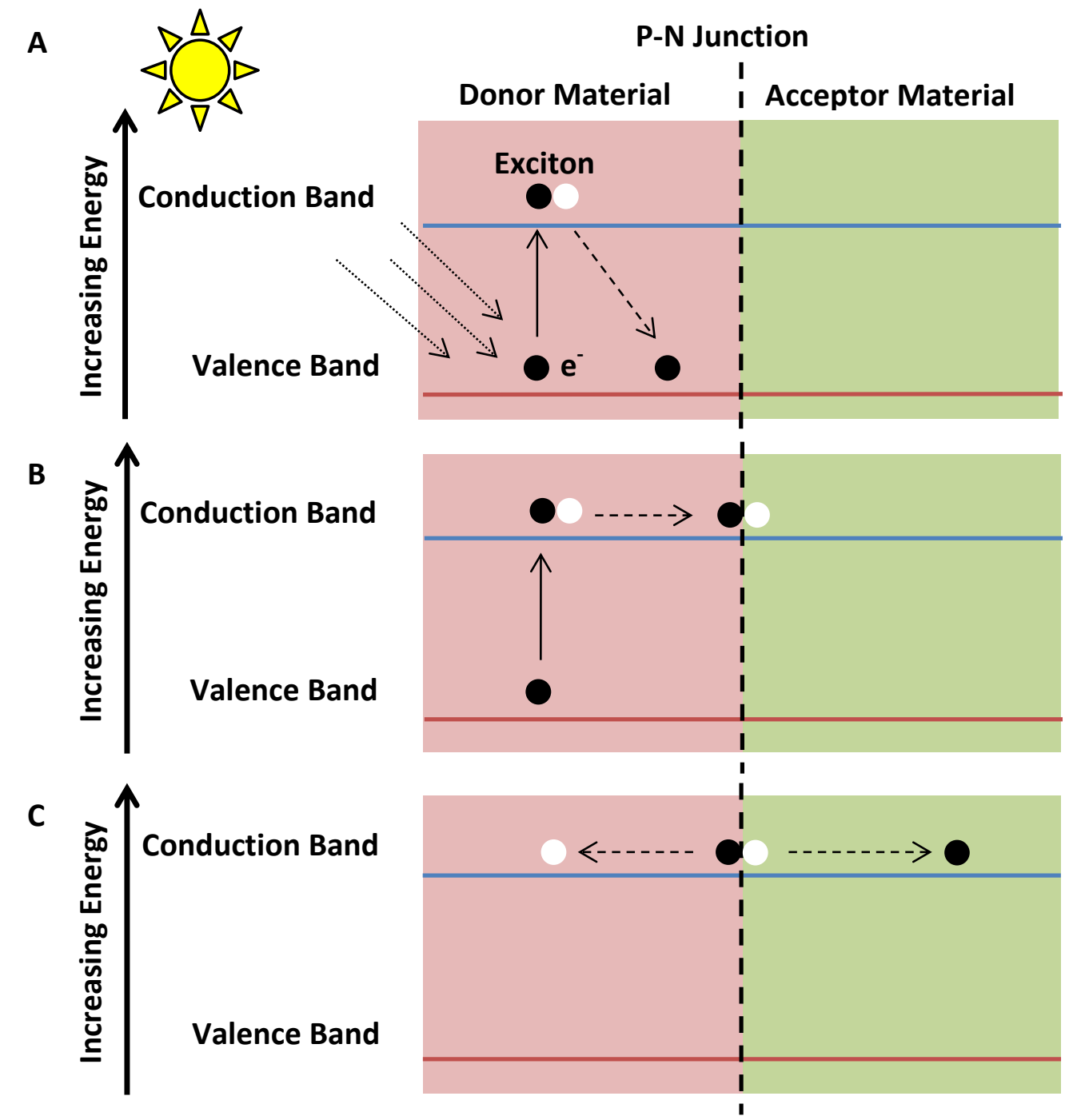

Figure 1.3: The photovoltaic effect is the phenomenon responsible for the generating current in solar cells. It involves the generation of excitons by incident light, which can then either decay (a) or diffuse to the donor/accepter interface of the $p-n$ junction. Following this, local fields split the exciton into its constituent charge carriers, which are then free to propagate through the device until they reach their respective electrodes. 
Although it would theoretically be possible to create a strong electric field across the entire device by applying an external electric potential, this is less than ideal because the power required to apply such a potential would likely surpass the power the device would produce. Fortunately, strong but short range local electric fields may be created simply by juxtaposing two materials with differing electron affinities. In traditional inorganic solar cells, this interface commonly referred to as a p$\mathrm{n}$ junction. In the case of organic photovoltaics, the material with the higher electron affinity (n-type semiconductor) is termed the acceptor material because it accepts electrons, and the material with the lower electron affinity ( $p$-type semiconductor) is termed the donor material because it donates electrons. Because of the small effective range of these local electric fields, the excitons must diffuse to the interface between the two materials, where the local fields are the strongest, in order to be split (Figure 1.3c). Once the exciton has been split, the charge carriers may then be conducted to the electrodes, with the electrons traveling through the acceptor material towards the anode and the holes traveling through the donor material towards the cathode.

The organic nature of CPs imparts several major advantages to polymer solar cells. Unlike the crystalline silicon used in traditional solar cells, semiconducting polymers are generally flexible, allowing for the 
creation of flexible solar cells. Additionally, as organic molecules, CPs are typically soluble in a number of organic solvents, meaning that polymer solar cells may be prepared using solution based processing techniques. Solution processibility is a particularly desirable trait, as solution based processing techniques are low cost and high volume, especially when compared to the expensive, high-tech vapor deposition techniques required to create traditional crystalline silicon solar cells ${ }^{9}$. Furthermore, semiconducting CPs are much cheaper than crystalline silicon and have higher absorptivity constants (less $\mathrm{CP}$ is required to gather the same amount of light), resulting in much lower overall device costs for polymer solar cells.

Unfortunately, polymer solar cells have some major drawbacks, as well. Organic solar cells typically suffer from relatively low power conversion efficiencies (PCEs): they generally produce less power than other, more established types of solar cells. The record for efficiency with a polymer solar cell in a research setting is just above $10 \%$ while most other types have efficiencies well over $15 \%$, with the record for crystalline silicon being over $25 \%$ in a research setting (Figure 1.4) and approaching $20 \%$ in an industrial setting (devices which are actually produced to be sold $)^{10}$. This means that the BEST polymer solar cells now currently being produced are a little less than half as efficient as the crystalline silicon 
solar cells which have already entered industrial production. Furthermore, because the CP active materials are conjugated organic molecules, they are particularly sensitive to oxidative degradation and UV light. This is somewhat of a problem for devices which are designed to operate in direct sunlight for years at a time; unsurprisingly, polymer solar cells generally exhibit relatively low operational lifetimes.

\subsection{Improving Operational Lifetimes of Polymer Photovoltaic Devices}

Although polymer photovoltaic devices do have several extremely promising advantages, they are not currently viable because of their low PCE and short operational lifetimes. It is commonly accepted in the field of polymer photovoltaics that to be successful, polymer solar cells must be able to achieve 10 years of operational lifetime at $10 \%$ PCE. As such, improving the lifetime and efficiency of polymer solar cells to meet these goals is currently the primary focus in the field of polymer photovoltaics. There are many different methods which have been employed to attempt to accomplish these goals. Most of the efforts to improve efficiency have been focused on increasing the dissociation of generated excitons or improving the mobility of the charge carriers ${ }^{12}$, while improvements to lifetime have focused mainly on preventing the oxidation of organic active materials through improving electrodes and device packaging ${ }^{13}$. 


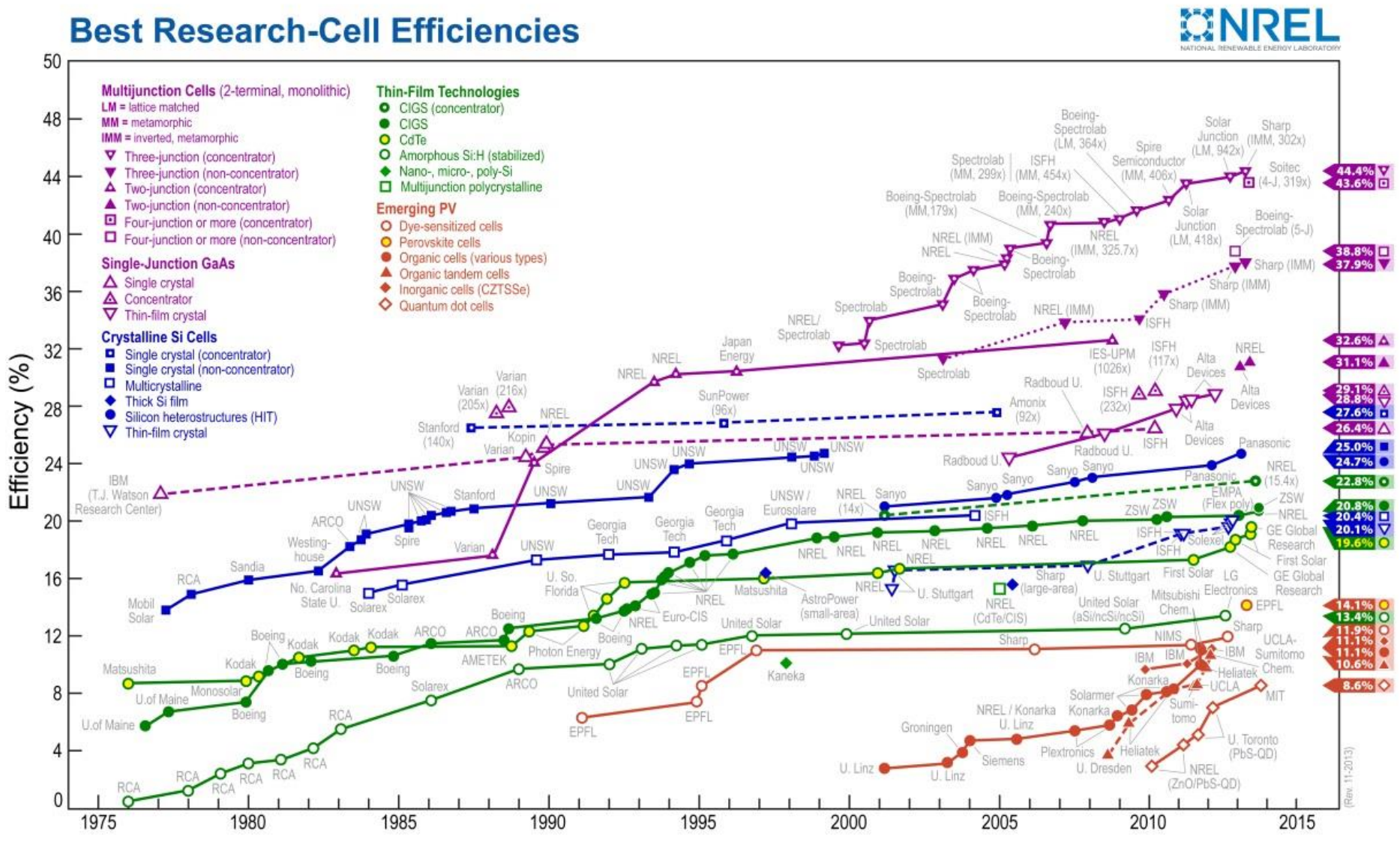

Figure 1.4: Efficiency records for various photovoltaic cells produced by research groups, organized by material type ${ }^{11}$. 
The causes of low operational lifetime in polymer solar cells are varied and although many of them are easily solved, the sheer number of problems can be an issue. As do most polymers, CPs can undergo free radical oxidative degradation in the presence of UV light (Figure 1.5) ${ }^{14}$. Similarly to the generation of excitons in the photovoltaic effect, the first step of photoinitiated oxidation, or photodegradation, involves light activating the polymer structure, causing an electron to become excited. However, in photodegradation, the incident photon (typically in the UV range of light, between $10-300 \mathrm{~nm}$ ) has a high enough energy to cause photolysis, the cleaving a chemical bond. Not only does this directly damage the polymer structure, but it also results in the formation of a pair of free radicals. Free radicals are atoms or molecules containing incomplete valence shells (unpaired electrons), making them highly reactive. After generation, these free radical species will propagate rapidly through the surrounding area, resulting in complex intrapolymeric reactions including chain splitting, side group scission and depolymerization, as well as interchain crosslinking ${ }^{15}$. These reactions create further defects in the polymer backbone, decreasing conductivity and decreasing device performance in polymer electronic devices. Furthermore, if oxygen is present, the polymer free radicals can react with it to form hydroxide free radicals, which are even more reactive and mobile and can cause major degradation. 


\section{Photolysis}

Absorption and excitation of binder or other film components

Energy dissipation and bond cleavage with formation of free radicals

\section{Autoxidation}

Peroxy radical formation

Peroxy radical attack on polymer chain with hydrogen abstraction to form hydroperoxide and free radical

Fragmentation of hydroperoxide by ultraviolet radiation

\section{Embrittlement}

Interchain cross linking between free radicals in adjacent chains and eventual embrittlement
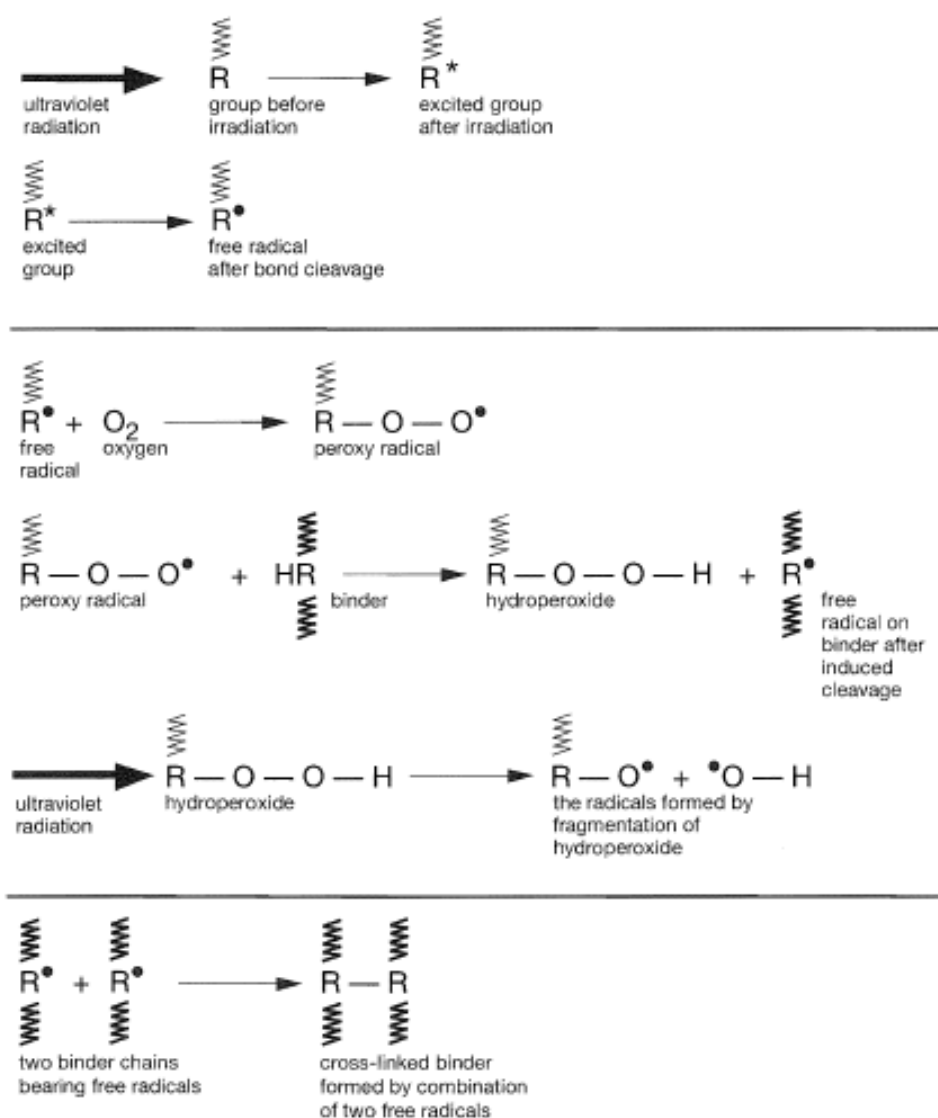

Figure 1.5: Photoinitiated oxidation is a major concern for all polymer coatings.

UV initiated photodegradation is a problem in most polymer materials, but can be particularly damaging in CP based photovoltaic devices. Although the highly conjugated nature of CPs infers low absorptivity in the UV range, over a long time frame the generation of radicals is significant and degradation occurs ${ }^{9}$. One approach to this problem is to use a UV filtering coating to prevent UV light from reaching the cell ${ }^{16}$. Unfortunately, this has shown limited success in retarding the rate of degradation. In other polymer based films, such as paints, free 
radical photodegradation is prevented using free radical stabilizing additives like hindered amine light stabilizers, but these additives may negatively affect the conductivity and film morphology of CP films, thus reducing device efficiency. Another common strategy used to reduce UV degradation in paints involves the addition of inorganic UV absorbers into the film such as zinc oxide. This approach has been utilized with some success in CP solar applications: certain UV absorbing inorganic particles may actually be useful as active components of the solar cell, and this category of solar cells will be discussed later.

Another issue reducing the lifetime of $\mathrm{CP}$ based polymer cells is their sensitivity to oxygen and water. Many CPs have low ionization potentials, which allows oxygen or water to form charge carrier complexes with the polymer backbone, causing oxidation ${ }^{13}$. Oxygen and water are often introduced in small quantities during device construction, and may even diffuse through pinholes in the electrodes ${ }^{17}$. Because of this, it is important that the devices be very well sealed under inert atmosphere so as to limit the introduction of these oxidative species. The electrodes themselves can also cause oxidation: certain metals which are commonly used as electrodes (such as aluminum, a common cathode) produce substantial degradation of the organic active material, reducing efficiency ${ }^{18}$. In order to solve this issue, interfacial layers of more stable 
material may be coated onto the electrode in question, producing a passivating layer which prevents degradation.

\subsection{Improving Efficiencies of Polymer Photovoltaic Devices}

Increasing the efficiency of polymer solar cells is a complicated problem with many different strategies being employed to combat different efficiency losses. One of the major sources of losses in polymer solar cells involves the generation and utilization of excitons. As mentioned, photovoltaic cells rely on the strong local fields present at $p-n$ junctions to dissociate excitons into electrons and electron holes. However, if the exciton cannot reach this interface quickly enough, it recombines and can no longer be used to generate current. This is somewhat of a nonissue for many inorganic semiconductors: in crystalline silicon, for example, excitons may last as long as 1 millisecond and are able to travel hundreds of micrometers ${ }^{19}$, which allows for relatively large device features. However, exciton diffusion lengths in polymers are much smaller, typically around $10 \mathrm{~nm}^{20}$. These small diffusion lengths necessitate extremely small device features: in order to maximize exciton dissociation, any exciton generated must be able to reach an interface within $10 \mathrm{~nm}$. As such, the most common polymer device structure involves thorough mixing of the donor and acceptor materials, resulting in a randomly dispersed 
donor/acceptor arrangement, commonly termed a Bulk HeteroJunction $(\mathrm{BHJ})$ arrangement (Figure 1.6b).

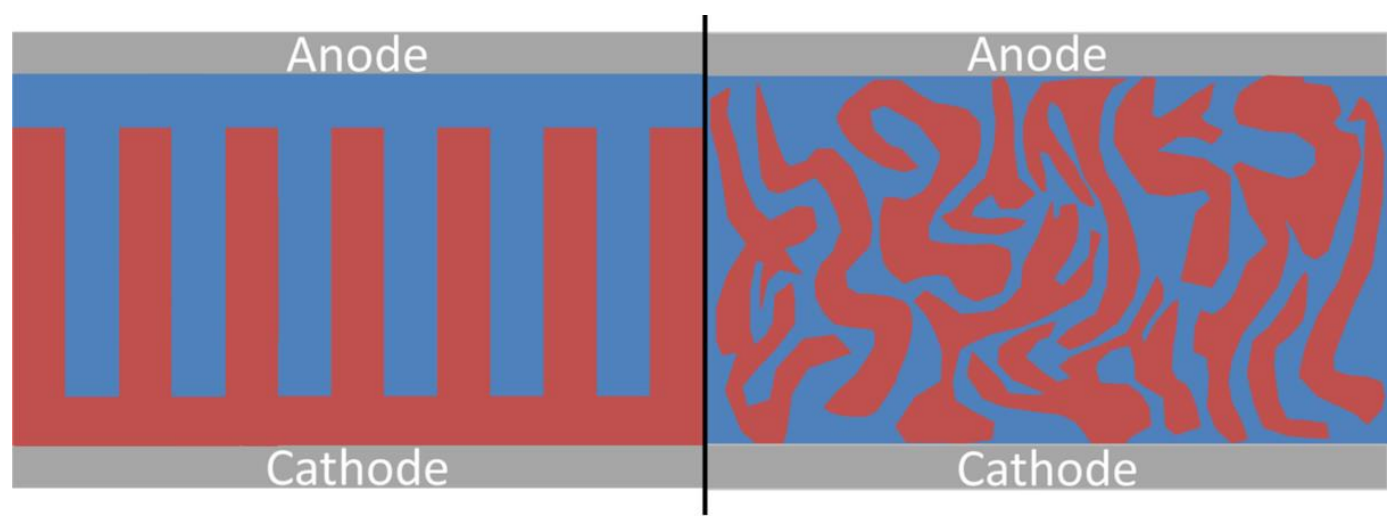

A) Ordered bulk heterojunction

B) Bulk heterojunction

Figure 1.6: A bulk heterojunction device model (b) allows for increased interfacial area but results in nonideal charge pathways. The nanoscale periodicity of the ordered bulk heterojunction model (a) solves this issue while maintaining high interfacial area.

Although the $\mathrm{BHJ}$ arrangement does improve exciton splitting, the inherently random nature of the donor/acceptor mixture is problematic. Often, there will be "islands" of donor or acceptor material that do not connect to the electrodes, which results in the generation and trapping of charge carriers inside these islands. Additionally, the randomness of the $\mathrm{BHJ}$ arrangement will still generate some domains with dimensions over $10 \mathrm{~nm}$, resulting in further reductions in exciton dissociation. The ideal device arrangement is one in which the donor and acceptor domains are 
precisely controlled at the nanoscale, allowing for the creation of very regular, nanosized domains arranged in a densely packed vertical array (Figure 1.6a). This Ordered $\mathrm{BHJ}(\mathrm{OBHJ})$ arrangement has been the subject of intense research, as it is widely thought to be one of the key breakthroughs needed for polymer solar cells to be commercially viable.

Another area of focus in polymer photovoltaics involves the improvement of charge transport efficiency in the polymer active layer. The relatively low mobility of charge carriers (specifically holes) in polymer films is often attributed as one of the primary causes for the reduced efficiencies seen in polymer photovoltaic devices ${ }^{21}$ : low charge carrier mobility is associated with increased exciton recombination and decreased current flow ${ }^{22}$. One of the simplest ways to mitigate this problem is to improve the conduction mechanism utilized by the polymer films.

Because the main mode of conduction in CPs is through the pi bonds in the polymer backbone, $\mathrm{CP}$ chains are often thought of as analogous to wires: charge carriers move much more quickly parallel to the direction of the polymer backbone than perpendicular ${ }^{23}$. Ideally, charge carrier conduction would be exclusively parallel to the polymer backbone, but realistically this is not the case: charge carriers must be able to "hop" between chains as well as conduct along them in order for 
current to be produced over any substantial distance ${ }^{24}$. Chain hopping occurs most readily with highly aligned polymer chains: that is to say, polymer films with large crystals and few defects/grain boundaries. Unfortunately, defects in polymer crystallinity are unavoidable as no completely crystalline polymers exist. Covalent orientational restrictions make the incorporation of amorphous domains in polymers inevitable, disrupting long range crystallinity. However, maximizing polymer alignment is still crucial, as crystalline morphologies have been widely observed to significantly impact the efficiency of CP photovoltaic devices $^{25}$.

Beyond inter-molecular alignment, CP intramolecular conformation (the shape of the backbone) also has substantial effects on the polymer's conductivity, and thus on polymer photovoltaic performance ${ }^{26}$. Because conduction occurs in CPs as a result of overlapping pi orbitals, CPs are most conductive when the backbone adopts an idealized rigid, linear conformation which allows for the pi orbitals to be geometrically aligned. In this case, we can model the polymer as a one-dimensional electron pathway and apply the idealized 1-D particle in a box equation to them:

$$
E_{n}=\frac{n^{2} h^{2}}{8 m L^{2}}
$$

in which $E_{n}$ is the energy of the electron, $n$ is an integer corresponding to the energy level of the electron, $h$ is Planck's constant, $m$ is the mass of 
the electron and $L$ is the length of the theoretical one-dimensional box. In this case, the "box" is the linear, rigid portion of the polymer, so the length of the box, $L$, is the conjugation length of the polymer.

Because the band gap is the difference energy between the HOMO (energy level $n=x$ ) and the lowest unoccupied energy level (energy level $n=x+1$ ), the band gap for this idealized model is entirely dependent upon the conjugation length. As the conjugation length increases, the band gap decreases, increasing the conductivity. In this idealized scenario where the polymer backbone is perfectly linear, the conjugation length is dependent only on the length of the backbone (i.e. the molecular weight). However, realistically there will be bends and twists in the polymer backbone, resulting in a decrease in practical conjugation length as the linear portion of the polymer is smaller than the total chain length. This reduction in effective conjugation length (ECL) increases the band gap of the $\mathrm{CP}$, causing a reduction in $\mathrm{CP}$ conductivity. As such, one key area of research in the field of polymer solar cells involves the control over CP backbone conformation.

\subsection{Controlling Polymer Alignment and Crystallinity}

The ability to control $\mathrm{CP}$ conformation and crystallinity is essential to optimize polymer solar cell performance. Generally, increased inter-chain crystallinity and effective conjugation length $(E C L)$ results in increased $C P$ 
conductivity, which improves charge carrier mobility in the system, and thus device PCE. The conformation of the $\mathrm{CP}$ is controlled by the interactions between the polymer and the solvent, and thus may be influenced through solvent selection. In the case of poly(3-hexylthiophene) (P3HT), one of the most common CPs used in photovoltaic applications, solvent interactions can determine whether the polymer adopts the more rigid, linear rod conformation or the relaxed nonlinear coil conformation (Figure 1.7).

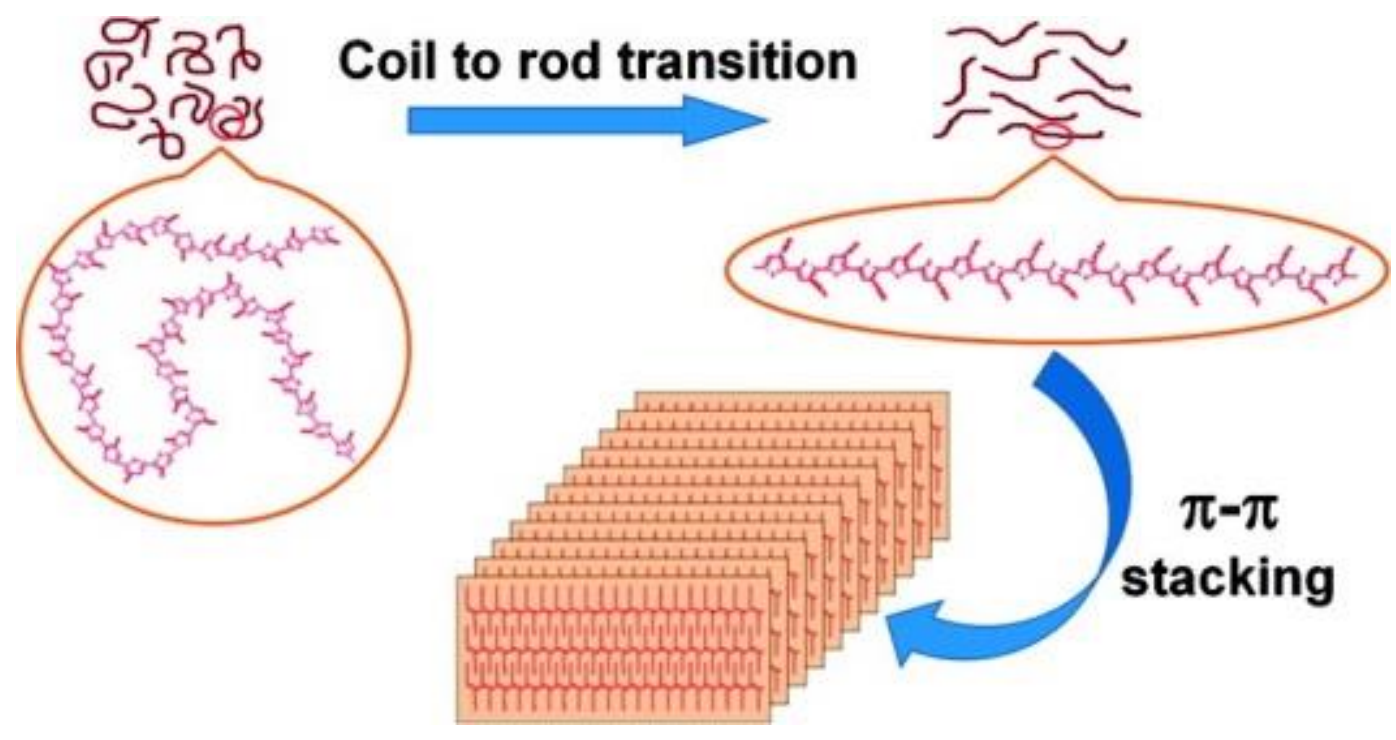

Figure 1.7: Solvent interactions influence the conformation of the polymer. Shown here, P3HT adopts the more rigid rod conformation in poor solvents (right) and a relaxed coil conformation in good solvents (left) ${ }^{27}$.

When P3HT is in a good solvent, the favorable polymer-solvent interactions dominate, producing well solvated coiled polymer 
conformation. However, when $\mathrm{P} 3 \mathrm{HT}$ is dissolved in a poor solvent, the intrapolymer interactions dominate, particularly polythiophene ring pi-pi stacking interactions. This causes the polymer to adopt a rigid rod shaped conformation, which can aggregate with other rod conformation polymer, crystallzing to reduce solvation ${ }^{27-28}$. These rod structures are highly linear and the pi-pi stacking interactions result in increased ECL, and thus increased conductivity ${ }^{29}$. Unfortunately, if the solvent is too poor, the polymer will precipitate out of solution, preventing uniform film formation. Thus, optimizing performance involves the selection of a solvent which is poor enough to induce rigid crystallites but not so poor that it causes precipitation. Additionally, the solvent must have the appropriate physical and rheological properties, such as vapor pressure and viscosity, for the desired coating method which will be used to form the polymer film.

Another common factor used to influence polymer conformation and crystallization is temperature. $\mathrm{P} 3 \mathrm{HT}$, for example, may exhibit the rod conformation in a given solvent at lower temperatures, but adopt a coiled conformation if the temperature is increased past a critical temperature ${ }^{30}$. Because of the macromolecular nature of $\mathrm{CPs}$, the final conformation and crystallinity is determined by both thermodynamic and kinetic factors. The covalent bonds linking the monomer segments impart a conformational dependence upon the polymer: a change in position or orientation in one segment of a polymer chain will affect the position and orientation of the 
rest of the chain, as well as that of the chains surrounding it. This means that even though polymer crystallization is a thermodynamically favorable process under the right conditions, orientational constraints arising from the interconnected nature of the monomer units of the polymer backbone interfere with the formation of a well aligned crystal structure. Because of this, polymer films tend adopt the greatest alignment when they are allowed to crystallize very slowly, with all of the chains having enough time to completely crystallize and align.

Traditionally, studies focused on polymer crystallization and conformation have been primarily performed using differential scanning calorimetry $(\mathrm{DSC})^{31}$, a method which measures the heat capacities of samples subjected to constant increases and decreases in temperature. However, DSC is time consuming and cannot measure the effects of solvents on polymer conformation in solution. Because of the propensity of CPs to absorb visible light, in many cases it is possible to determine the conformation of a CP through spectral analysis. In particular, the conformational transitions and crystallization of substituted polythiophenes such as P3HT have been studied using UV-visible spectrophotometry ${ }^{28,32}$, because the absorbance spectrum is highly dependent both upon the polymer backbone conformation and the pi-pi interactions associated with polymer alignment. 


\subsection{Hybrid Solar Cells}

Although there is much improvement to be made in the optimization of polymer morphology for solar applications, there are many other promising areas which are being explored to try to improve polymer solar cells. Hybrid solar cells are an example of this, having received major attention in recent years because of their potential to improve both lifetime and efficiency beyond the 10 year/10\% goals.

Hybrid photovoltaic devices incorporate crystalline semiconducting inorganic nanoparticles into the CP matrix, where they may act as an acceptor material. The reasoning behind this is that many semiconducting inorganic nanomaterials have very good optoelectronic properties, often with optical bandgaps in the visible or near-infrared range ${ }^{33}$. They are often much better electron conductors than the organic small molecules traditionally used as electron acceptors in polymer solar cells, such as Phenyl-C61-butyric acid methyl ester (PCBM) ${ }^{34}$. This is further enhanced by the fact that hybrid solar cells utilize single crystalline nanoparticles, which allows for particularly high electron mobility since the electrons don't have to cross crystal grain boundaries. When these crystalline nanoparticles are anisotropic (typically cylindrical), they also allow for direction of the electron flow throughout the device as the pseudo onedimensional arrangement of these small diameter cylindrical nanocrystals 
causes electrons to travel much more quickly parallel to the axis than perpendicular. This anisotropy also produces an opportunity to easily create an ordered $\mathrm{BHJ}$ nanostructure if the orientation of these anisotropic crystalline nanomaterials can be influenced from solution ${ }^{35}$. Inorganic materials also typically have very high electron affinities, and thus may increase the exciton dissociation in the device. There are a wide variety of nanomaterials which have been utilized in hybrid solar cells, including CdSe quantum dots/rods ${ }^{36}, \mathrm{TiO}_{2}$ nanorods ${ }^{37}$, carbon nanotubes ${ }^{38}$, and ZnO nanowires ${ }^{35 b, 39}$.

Although inorganic materials generally have good electron mobility and high electron affinities, hybrid solar cells require the use of nano-sized particles in order to optimize exciton collection. These nanomaterials, which are defined as objects having at least one dimension between 1$100 \mathrm{~nm}$, must be small enough to allow any generated exciton to be able to diffuse to the material boundary, where it may be dissociated. Many nanomaterials used in hybrid solar applications have diameters smaller than $30 \mathrm{~nm}$, which helps accomplish this. Furthermore, the smaller the size of the nanoparticle, the higher the surface area to volume ratio (demonstrated in Figure 1.8), which theoretically increases the amount of interfacial area available to split excitons for a given volume of material. Unfortunately, this effect also makes it very difficult to disperse small nanomaterials in the polymer matrix. Most nanomaterials have a very high 
surface energy and do not interact very favorably with low surface energy polymers ${ }^{40}$. This relative immiscibility is further exacerbated by the high surface area of nanomaterials, making unfavorable surface interactions a dominant force in determining film morphology. Because of this, nanoparticle aggregation is a serious problem in hybrid solar cells. This aggregation reduces the overall interfacial surface area available to split excitons, thus reducing device efficiency ${ }^{40}$. Furthermore, the polymer may also dewet from the surface of the nanoparticles, leaving air voids which damage film integrity and reduce device performance. Paints and other nanoparticle-containing polymer based coatings typically solve these problems through the use of dispersing additive, but these additives may not be suitable for use in solar cells because of negative effects on device performance. Because of these issues, hybrid core-shell nanocomposites are often used in place of unfunctionalized nanoparticles.

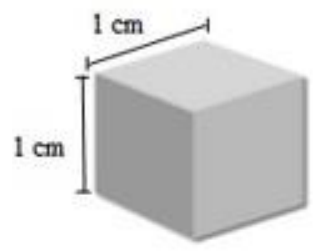

Total surface area: $6 \mathrm{~cm}^{2}$

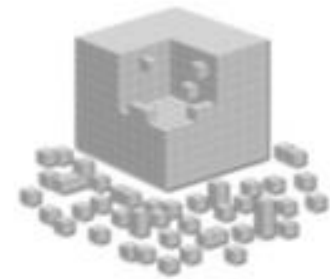

Total surface area: $60 \mathrm{~cm}^{2}$ (All $1 \mathrm{~mm}$ cube)

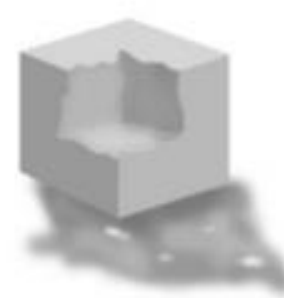

Total surface area: $60,000,000 \mathrm{~cm}^{2}$ (All $1 \mathrm{~nm}$ cube)

Figure 1.8: The surface area to volume ratio for a given particle increases exponentially as particle size decreases ${ }^{41}$. 


\subsection{Hybrid Core-Shell Nanocomposites}

Core-shell nanocomposites are nanoparticles which have had a shell of polymer attached to them before addition into a polymer matrix (Figure 1.9), and are analogous to nanoscale BHJs. Frequently, they are constructed using anisotropic, rod- or wire-shaped nanoparticles, resulting in pseudo one-dimensional (1D) hybrid nanomaterials. These nanocomposites have significantly lower surface energy than the unfunctionalized nanoparticles, making it much simpler to produce well dispersed, uniform films ${ }^{42}$. This approach also has the added benefit of enabling direct control over the polymer/nanomaterial interface, allowing for the optimization of structure so as to produce efficient interfacial electronic transport. Furthermore, these 1D hybrid nanomaterials have shown the propensity to spontaneously develop lyotropic liquid crystalline domains in solution ${ }^{35 a}$. This allows for the easy creation of organized, aligned arrays of $\mathrm{BHJ}$ nanostructures through the application of magnetic fields, a promising step towards solution processed OBHJ devices. 


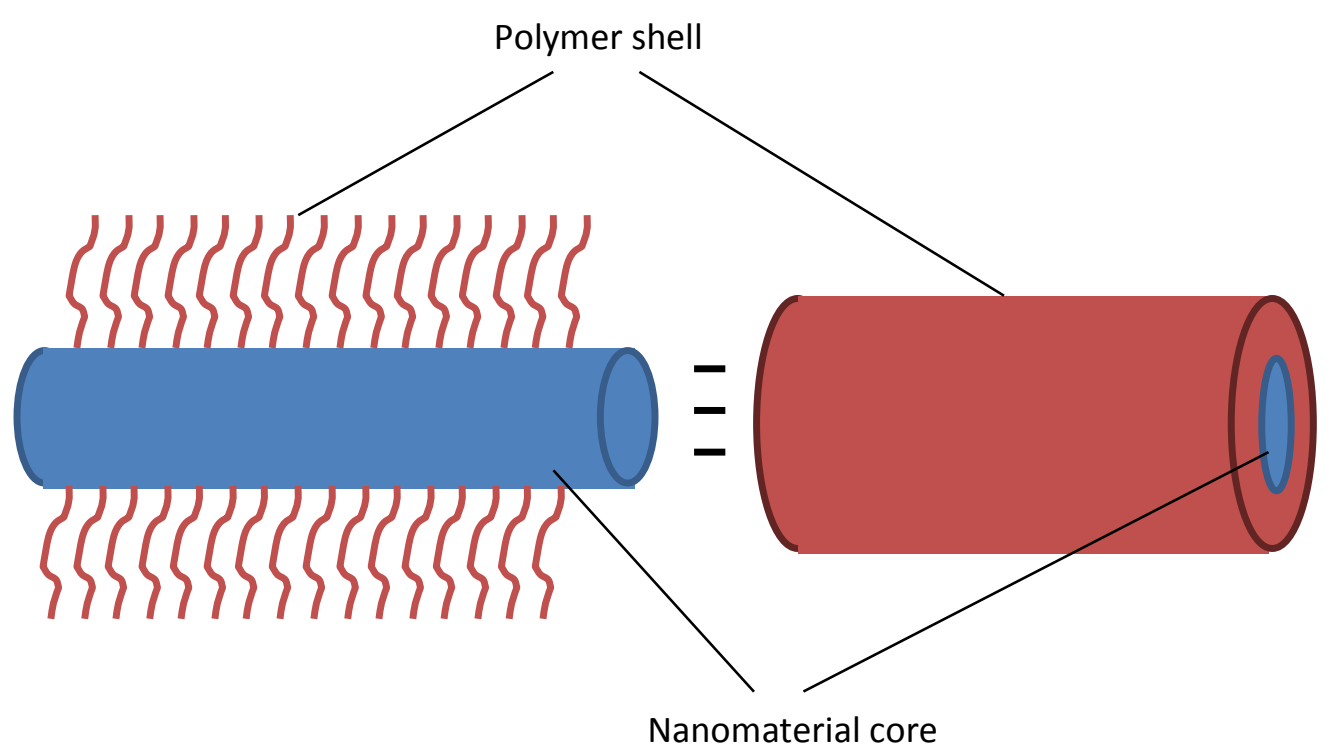

Figure 1.9: Core-shell nanocomposites consist of a nanomaterial core attached to a surrounding polymer shell.

1D core-shell nanocomposites have been produced through a variety of methods including the use of chemical linking agents to bind the polymer to the nanoparticle ${ }^{38 b}$, ligand exchange 34 , 43, direct polymerization of the CPs (growing the CP directly onto the nanoparticle surface) ${ }^{44}$, and the direct attachment of CPs using functional side/end groups ${ }^{45}$. Currently, the most commonly used approach is the direct attachment of end-functionalized CPs, in which the CP shell is anchored to the nanoparticle surface using functionalized polymer chain ends. The direct attachment method maximizes the strength of the local fields by keeping the donor and acceptor materials in intimate proximity, thus improving exciton splitting. However, end-functionalized attachment may 
decrease the efficient attachment of the polymer onto the nanoparticle because of the limited number of functional groups which may mediate attachment to the nanoparticle. Furthermore, this approach has been shown to result in the polymer adopting hairpin folding along the backbone ${ }^{39 b}$ (Figure 1.10). This hairpin folding means that coaxial charge carrier transport in the polymer must occur through a hopping-mechanism, because conductive transport may only occur along overlapped $\mathrm{p}$-orbitals of the backbone. Hopping charge transport is substantially slower than conductive transport, resulting in low overall conductivity in the polymer layer. Because of these problems, end-functionalized polymer grafting is not ideal.
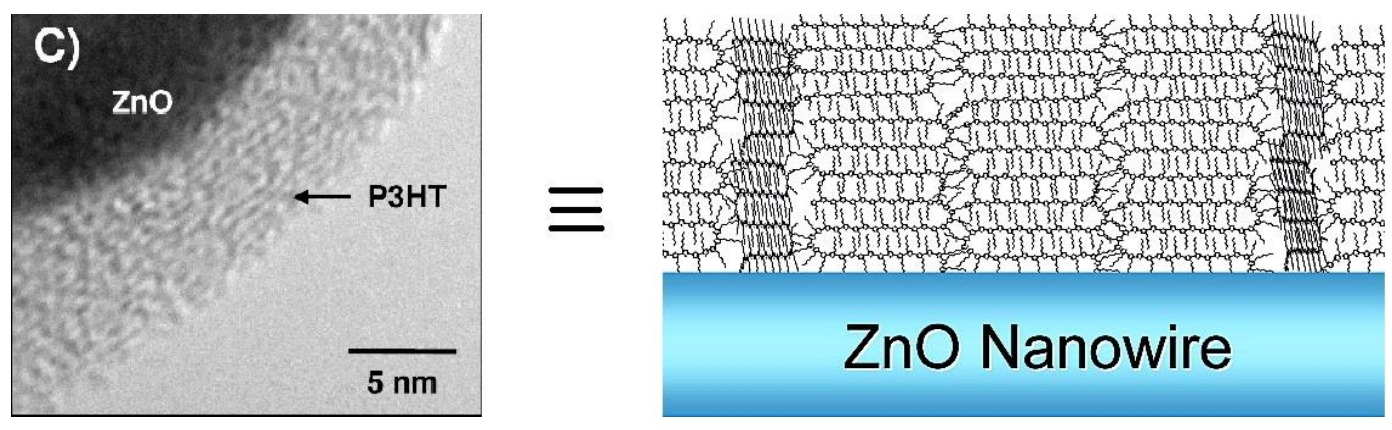

Figure 1.10: End-on polymer shell attachment results in hairpin folding of the polymer backbone, producing a torturous charge carrier pathway through the polymer shell ${ }^{39 b}$.

An improved attachment method would be one in which the polymer backbones are oriented parallel along the axis of the 
nanoparticle, such that parallel charge conduction could occur. Recently, core-shell nanocomposites have been produced with side-functionalized attachment through the use of ionic linking molecules and CP with functionalized side chain groups ${ }^{35 b}$. Although the use of ionic linking molecules incorporates an additional efficiency reducing interfacial layer, the side-on attachment method was seen to produce a polymer shell with highly extended backbone conformations, which should induce increased conductivity.

\subsection{Research Plan}

There are two major focuses of this work. The first is to characterize the conformational transitions and crystallization of carboxylic acid side-chain functionalized polyalkylthiophenes in dilute solutions. Parameters which dictate the transitions are also studied, including solvent, concentration, side-chain length and temperature. These findings will allow a better understanding of how to control the alignment and conformation of CPs in order to optimize film morphology, which is one of the key criteria for producing highly efficient polymer solar devices.

The other major focus is the formation of an anisotropic core-shell nanocomposite with direct chemical core-shell linkages by directly grafting a P3HT derivative with carboxylic acid functionalized side-chains onto 1D $\mathrm{ZnO}$ nanowires, produced using a solvothermal synthetic method. The 
formation of the nanocomposite is verified and the effects of grafting on polymer conformation and crystallinity are investigated. The effects of synthetic conditions (i.e. solvent, reaction time) on the nanocomposite are also investigated. 
2. Methods and Materials

\subsection{Material preparation}

2.1.1. Preparation of Zinc Oxide Nanowires (ZnO-NW) $)^{35 a}$

Zinc nitrate hexahydrate $\left(\mathrm{Zn}\left(\mathrm{NO}_{3}\right)_{2} \cdot 6 \mathrm{H}_{2} \mathrm{O}\right.$, Fisher Scientific), sodium hydroxide ( $\mathrm{NaOH}$, Sigma Aldrich), ethanol (EtOH, Sigma Aldrich) and ethylene diamine (EDA, Sigma Aldrich) were used as received from chemical suppliers. The $\mathrm{Zn}\left(\mathrm{NO}_{3}\right)_{2}$ was received as translucent white crystals, the $\mathrm{NaOH}$ was in opaque white pellets and the EDA and EtOH were clear liquids. In a typical procedure for the preparation of $\mathrm{ZnO}$ nanowires, a $2.0 \times 10^{-2} \mathrm{M}$ zinc solution was prepared by dissolving $0.888 \mathrm{~g}$ $\mathrm{Zn}\left(\mathrm{NO}_{3}\right)_{2} \cdot 6 \mathrm{H}_{2} \mathrm{O}$ in $150 \mathrm{~mL} \mathrm{EtOH}$ at room temperature, after which $3.6 \mathrm{~g}$ $\mathrm{NaOH}$ was added and completely dissolved with vigorous stirring under atmospheric conditions. The resulting solution was sonicated for 60 minutes using a Heat Systems Misonix Sonicator Ultrasonic Processor XL 2000, after which $15 \mathrm{~mL}$ of EDA was added, and the solution was sonicated for 30 minutes in a Branson 2510 bath sonicator. The system was then transferred to a Teflon liner and sealed in a stainless steel autoclave at $130{ }^{\circ} \mathrm{C}$ for 72 hours. Resulting zinc oxide nanowires were collected and purified by repeated washing with deionized water and ethanol, following which the specimens were dried in oven for at least 
three hours. The dried samples were ground into a fine white powder using a mortar and pestle and then stored.

\subsubsection{Preparation of Nanocomposites}

ZnO-NW were prepared as described, and used as a fine white powder. Regioregular ( 90\% head-to-tail) Poly[3-(5-carboxypentyl) thiophene-2,5-diyl] (P3CPenT, weight average molecular weight $\left(\overline{\mathrm{M}}_{W}\right)=$ $55-65 \mathrm{~kg} / \mathrm{mol}$, Rieke Metals Inc.) was used as received, as a coarse, clumped dark powder. In a typical procedure for the preparation of nanocomposites, the fine powder of $\mathrm{ZnO}$ nanowires $(10 \mathrm{mg})$ was dispersed in P3CPenT (2 mL, $5.0 \mathrm{mg} / \mathrm{mL}$ ) in pyridine (Sigma Aldrich) or dimethylsulfoxide (DMSO, Sigma Aldrich) and left to shake at 1250 RPM, room temperature. This allowed the carboxylic acid groups on the polymer side chains to react with the $\mathrm{ZnO}-\mathrm{NW}$, forming zinc-carboxylate bonds (Figure 2.1). Over the course of the reaction, the supernatant would become clearer, as the polymer reacted, and if the samples were allowed to react for a sufficient period of time, the supernatant would be completely clear. However, in a typical preparation procedure, the samples were allowed to react in a high concentration polymer solution for 2-4 weeks, and were removed before the supernatant was clear, yielding dark nanocomposite. Nanocomposite samples with significantly lower polymer loading could also be prepared using a lower polymer:ZnO-NW ratio. However, the low polymer loading made it difficult to characterize the 
polymer shell of these low loading nanocomposites, so for the purposes of this study, most characterization was performed on samples with between $30 \%$ and $50 \%$ polymer loading.

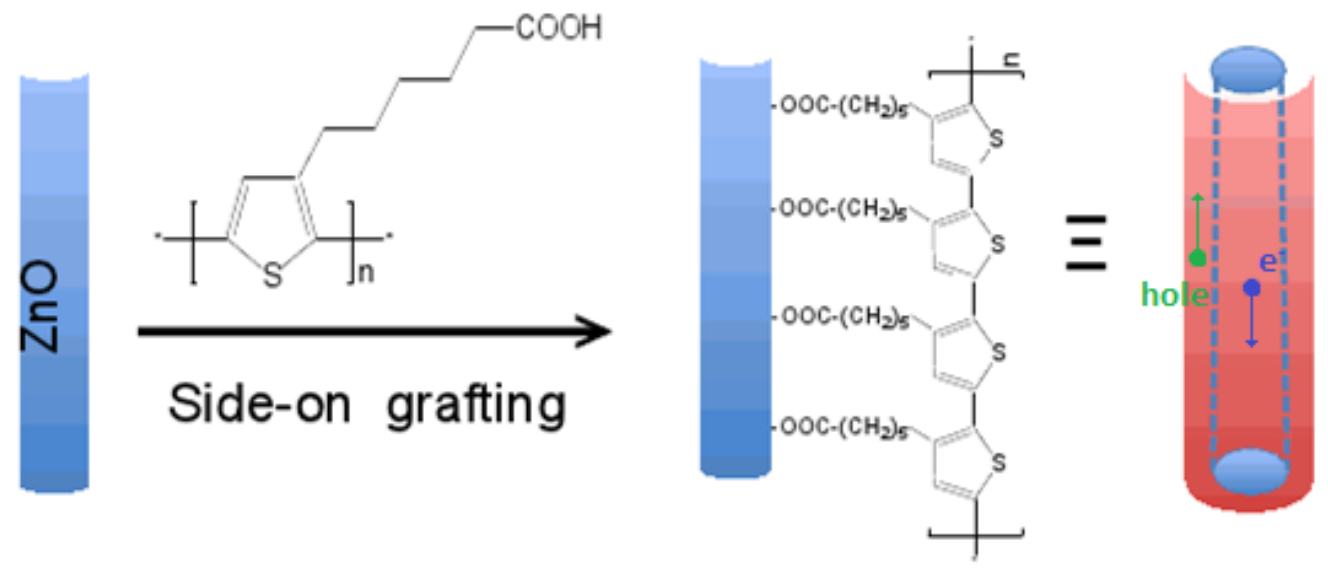

Figure 2.1: The side-on chemical grafting of the nanocomposite is made possible by the reaction of the carboxylic acid functional side-groups with the $\mathrm{ZnO}-\mathrm{NWs}$.

After reaction, microcentrifugation at 10,000 RPM (Centrifuge Biotechnical Services) produced separation. The remaining polymer in the supernatant was removed, the nanocomposite pellet was washed thoroughly with solvent to remove excess free polymer and the sample was rinsed with ethanol to enable more rapid drying. Afterwards, the colored nanocomposite precipitate was dried in an oven at $80{ }^{\circ} \mathrm{C}$ for 3 hours and then stored at room temperature in a sealed, dark environment. Stock dispersions of the nanocomposite were prepared by adding solvent to a measured amount of dry nanocomposite in a centrifuge vial, followed 
by sonication for 5-10 minutes or until the nanocomposite was dispersed. These stock solutions were then stored at room temperature in a sealed dark environment until measurement, immediately before which they would be resuspended by brief sonication.

\subsubsection{Preparation of Pristine Polymer Samples}

Regioregular ( 90\% head-to-tail) Poly[3-(5-carboxypentyl) thiophene-2,5diyl] (P3CPenT, average $\overline{\mathrm{M}}_{W}=55-65 \mathrm{~kg} / \mathrm{mol}$, Rieke Metals Inc.), regioregular ( 90\% head-to-tail) Poly[3-(5-carboxyhexyl) thiophene-2,5diyl] (P3CHexT, average $\overline{\mathrm{M}}_{W}=55-65 \mathrm{~kg} / \mathrm{mol}$, Rieke Metals Inc.), regioregular ( 90\% head-to-tail) Poly[3-(5-carboxybutyl) thiophene-2,5diyl] (P3CButT, average $\overline{\mathrm{M}}_{W}=55-65 \mathrm{~kg} / \mathrm{mol}$, Rieke Metals Inc.) and regioregular ( 90\% head-to-tail) Poly[3-(5-carboxypropyl) thiophene-2,5diyl] (P3CButT, average $\overline{\mathrm{M}}_{W}=55-65 \mathrm{~kg} / \mathrm{mol}$, Rieke Metals Inc.) were all used as received, with samples generally presented as clumpy or crystalline dark powders. Standard polymer solutions were created at 2.5 $\mathrm{mg} / \mathrm{mL}$ in DMSO or pyridine by adding $1.00 \mathrm{~mL}$ of solvent by micropipet to $2.5 \mathrm{mg}$ of solid polymer in a centrifuge vial. The solutions were heated in an oil bath to $75{ }^{\circ} \mathrm{C}$ until the polymer was dissolved, and then cooled to room temperature in a water bath for at least an hour. These samples were stored at room temperature in the dark for no more than two weeks. 


\subsection{Characterization and Analysis}

\subsubsection{UV-Visible Absorption Spectrophotometry}

UV-visible absorption spectra were taken in solution on a Jasco V550 spectrophotometer, which employed a sample temperature control fixture. All samples were prepared via serial dilution from stock solutions and were measured in glass cuvettes, sealed from ambient atmosphere with stirring. UV-vis absorption spectra were obtained for all nanocomposite samples to characterize the backbone conformation of the polymer shell. Additional measurements were performed on an extensive number of pure polymer solutions to characterize the conformational transition and crystallization processes of P3CATs. In all polymer spectra, the stock was diluted into the cell containing the appropriate solvent(s) at the appropriate temperature, and allowed to equilibrate for 30 minutes before measurement was performed. Similarly, for temperature ramps, the sample was allowed to equilibrate for 30 minutes at the initial temperature, and then ramped at a constant heating rate of $2{ }^{\circ} \mathrm{C} / \mathrm{min}$ to the final temperature.

For kinetics measurements, the stock solutions were heated to 80 ${ }^{\circ} \mathrm{C}$ to ensure that the polymer was in coil form, and then was diluted quickly into the cell containing solvent at the appropriate temperature in order to induce a rapid conformational transition. Absorbance $(\lambda=590 \mathrm{~nm})$ 
versus time plots were collected from these dilutions, and the change in absorbance over the first 5 seconds was taken as the initial rate. This time was selected because it was the highest time at which all curves could be modeled by linear functions with $\mathrm{R}$-squared values of greater than 0.90 . Following the determination of the initial rates, the method of initial rates was used to determine the order of the reaction and the rate constant.

\subsubsection{UV-Visible Emission Spectrophotometry}

Photoluminescence emission spectra were recorded in solution on a Jasco SP-6500 fluorometer equipped with a temperature control fixture. All samples were prepared from stock sample solutions and measured in unfrosted quartz cuvettes, sealed from atmospheric conditions using an excitation wavelength of $450 \mathrm{~nm}$. UV-visible emission spectra were collected for all nanocomposite samples to be compared to pure polymer samples to determine photoluminescence quenching activity in the nanocomposite. For this purpose, equal polymer concentrations were maintained in all measured samples, so that direct comparisons could be drawn between the emission activity of the nanocomposite compared to the pure polymer. The polymer concentration in the nanocomposite samples was determined using the loading of the nanocomposite as determined from thermogravimetric analysis. For the determination of solvent and temperature effects, pure polymer samples were prepared 
and allowed to equilibrate at the appropriate temperature/in the appropriate solvent for half an hour before measurement.

\subsubsection{Fourier Transform Infrared Spectroscopy}

Fourier transform infrared (FTIR) spectra were obtained using a Nicolet 380 FT-IR spectrometer in attenuated total reflection (ATR) mode at a resolution of $8 \mathrm{~cm}^{-1}$ (1000 scans). All nanocomposite samples were analyzed by FTIR to confirm chemical attachment of the carboxylic acid functionalized side chains to the $\mathrm{ZnO}-\mathrm{NW}$. Nanocomposite and pure polymer samples were recorded in bulk, dry powder form. Spin cast polymer films of $1 \mathrm{mg} / \mathrm{mL}$ polymer solution in both DMSO and pyridine were prepared on glass slides and evaporated at room temperature, and were also analyzed by FTIR for comparison.

\subsubsection{Thermogravimetric Analysis}

Polymer loading and thermal degradation were examined using thermogravimetric analysis (TGA) on a TA Instruments Q500. TGA samples were analyzed using heating rates between $10-20^{\circ} \mathrm{C}$ in both oxygen and nitrogen atmospheres, although only oxygen atmosphere was used for the determination of polymer loading. Samples were measured in bulk dry powder form both for the nanocomposite and the polymer, and were loaded into pre-tared aluminum pans rated up to $600^{\circ} \mathrm{C}$. The degradation temperatures were determined by identifying maxima in the 
first derivative of the heating curve (sample weight with respect to temperature), while the total polymer loading of the nanocomposites was determined by the percent of sample weight lost at $600^{\circ} \mathrm{C}$.

\subsubsection{Transmission Electron Microscopy}

The core-shell morphology of the nanocomposites was verified using transmission electron microscopy (TEM). For TEM observation, samples were prepared by dispersing the dried nanocomposites in ethanol using brief sonication (less than 5 minutes), following which the dispersion was transferred via micropipette to the holey carbon film on 300 mesh copper grids purchased from Electron Microscopy Sciences. FEI Tecnai G2 Sphera and Hitachi HF2000 microscopes were used to image individual nanowires.

TEM was also used to produce length and width distributions of the ZnO-NWs through quantitative measurement of nanowire dimensions. These size distributions were performed by imaging a large number of nanowires and sampling nanowires for which the length and width were resolved.

\subsubsection{X-Ray Diffraction}

X-ray diffraction (XRD) was used to characterize the crystal structure of the nanocomposite polymer shell and ZnO-NW. Diffraction 
patterns were captured for all samples using a reflection geometry Siemens D5000 diffractometer, with a $1.54 \mathrm{~A} \mathrm{Cu}$ Ka radiation source. Nanocomposite and ZnO-NW samples were analyzed in bulk, while pure polymer was analyzed in bulk form and as spin cast films of $1 \mathrm{mg} / \mathrm{mL}$ polymer solution prepared on glass slides and evaporated in an oven at $80{ }^{\circ} \mathrm{C}$. 
3. Results and Discussion

\subsection{Nanocomposite Characterization}

\subsubsection{Confirmation of Side-On Chemical Core-Shell Linkage}

The preparation of the $\mathrm{ZnO} / \mathrm{P} 3 \mathrm{CPenT}$ nanocomposite produced materials which were visually distinct both from the $\mathrm{ZnO}-\mathrm{NW}$ and the P3CPenT polymer samples. The nanocomposite material exhibited a powdery texture similar to that of $\mathrm{ZnO}-\mathrm{NW}$, but instead of the pure white coloration typical of $\mathrm{ZnO}-\mathrm{NW}$, the nanocomposite showed the darker purple coloration of P3CPenT polymer. Furthermore, the nanocomposite was insoluble in solvents which would dissolve pristine P3CPenT at all temperatures, suggesting that polymer functionalization was successful. Indeed, a comparison of the FT-IR spectra for the nanocomposite and the polymer samples (Figure 3.1) reveals a significant change in the character of the carbonyl side chain group for the nanocomposite compared to that for the pure polymer.

Pristine P3CPenT polymer films bearing carboxylic acid side groups $(-\mathrm{COOH})$ show a strong $\mathrm{C}=\mathrm{O}$ vibrational band at $\sim 1700 \mathrm{~cm}^{-1}$ in the carbonyl stretching regions, which is indicative of hydrogen bonded $\mathrm{C}=\mathrm{O}$ vibrations ${ }^{46}$. This peak position is consistent in both the DMSO and pyridine cast P3CPenT films, which would seem to suggest that the strong interchain interactions denoted by the hydrogen bonding are not solvent 
dependent. After the polymer was grafted onto the $\mathrm{ZnO}-\mathrm{NW}$, this hydrogen bonded $\mathrm{C}=\mathrm{O}$ vibrational band nearly disappeared while new COO bands were observed in the $1510-1630$ and $1370-1500 \mathrm{~cm}^{-1}$ regions, representing the asymmetric and symmetric COO vibrations, respectively. This shift is consistent with the formation of a covalent carboxylate-zinc bond ${ }^{47}$, confirming the desired side-on chemical linkage between the polymer and ZnO-NW. Metal oxide-carboxylate covalent bonding may be either monodentate or bidentate ${ }^{48}$, but $\mathrm{ZnO}$-carboxylate bonding is commonly accepted to be bidentate ${ }^{49}$. The nanocomposites also show some very slight $\mathrm{C}=\mathrm{O}$ character, which is likely the result of additional

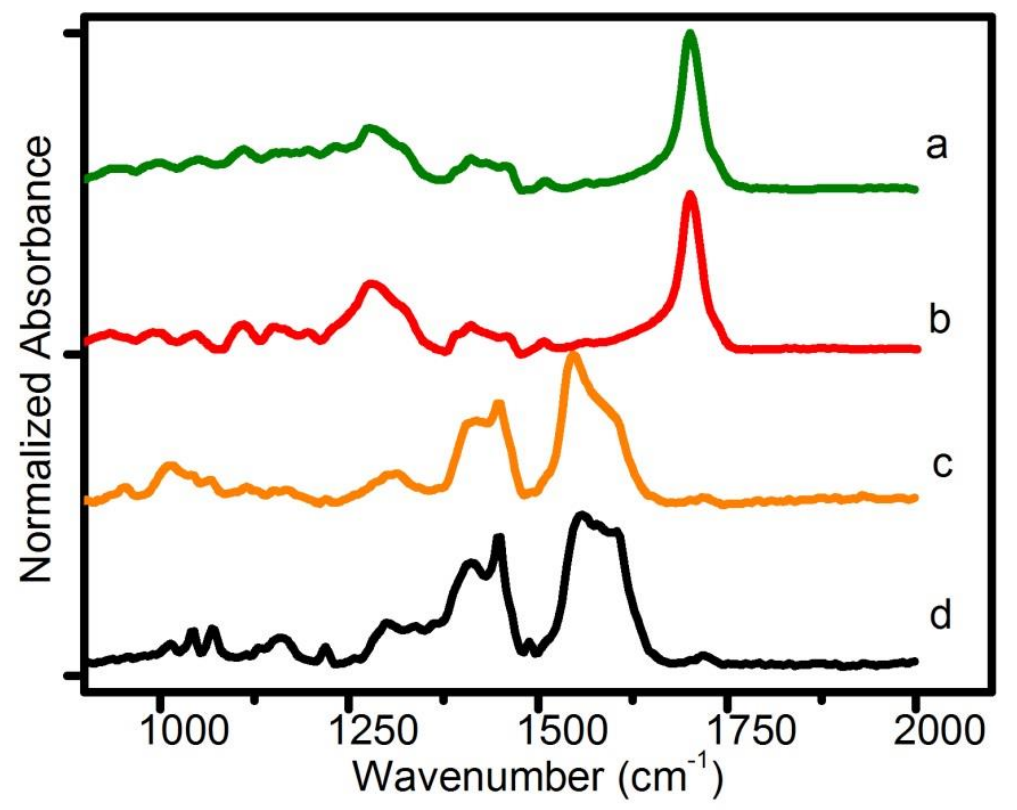

Figure 3.1: FT-IR spectra of pristine polymer films cast from pyridine (a) and DMSO (b) and dry bulk nanocomposite produced from solutions in pyridine (c) and DMSO (d) 
physical adsorption of P3CPenT polymer onto the nanowires past the first chemically grafted layer. The nanocomposite produced in DMSO also appears to exhibit a slightly broader asymmetric $\mathrm{COO}$ stretching vibration, which may indicate differences between the DMSO and pyridine samples in the $\mathrm{Zn}-\mathrm{COO}$ bonding.

The nanocomposite also exhibits a core-shell arrangement, as was expected. TEM imaging revealed no significant aggregations of nanowire structures, which, under high magnification, were found to have a polymer shell surrounding the $\mathrm{ZnO}-\mathrm{NW}$ core. The shell also appears to be somewhat nonuniform as a result of random physical adsorption of polymer, with some regions having very thick polymer shells $(10+\mathrm{nm})$ while others have a much thinner coverage $(2-3 \mathrm{~nm})$. This confirms that the nanocomposite has the capacity for significant physical adsorption of additional polymer past the first layer.
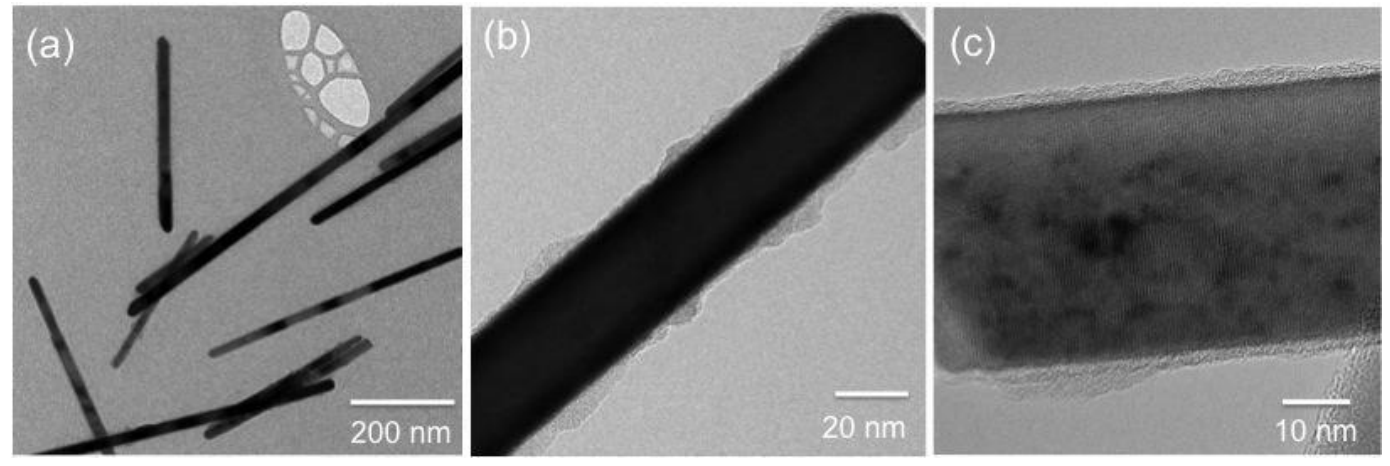

Figure 3.2: Low resolution (a) and high resolution (b, c) TEM images of hybrid nanocomposite samples. 


\subsubsection{Nanocomposite Thermal Characterization}

In light of the high thickness of the polymer shell, the nanocomposite was studied to determine the effects of grafting on the polymer. The polymer loading of the samples was seen to vary significantly with initial polymer concentration, as shown by TGA heating curves (Figure 3.3). This allows manipulation of the polymer loading as a means to optimize nanocomposite optoelectronic properties by changing the synthetic procedure, but also enables the construction of hybrid nanocomposites with high polymer loading, which is necessary in order to

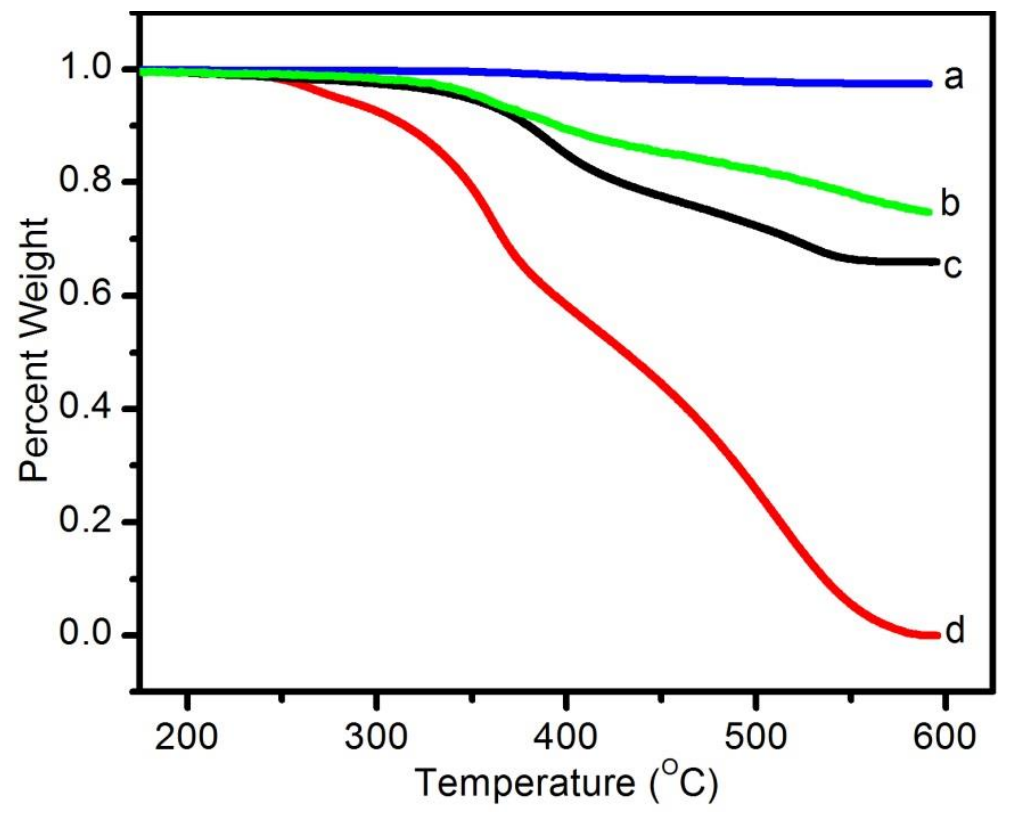

Figure 3.3: TGA heating curves for nanocomposite samples prepared in DMSO using P3CPenT:ZnO initial concentrations of 1:9 (a), 3:7 (b) and 1:1 (c), with bulk pristine P3CPenT polymer (d) as a reference. 
study the effects of grafting on the morphology, arrangement and conformation of the P3CPenT shell.

The nanocomposite polymer shell also appears to exhibit a thermal degradation mechanism which is different from that of pristine P3CPenT polymer, which can be clearly seen in a normalized comparison of the two TGA heating curves (Figure 3.4). Pristine P3CPenT polymer exhibits a multi-step degradation mechanism with a relatively minor initial decrease in weight seen at $265{ }^{\circ} \mathrm{C}$ followed by more significant secondary and tertiary degradations at $360{ }^{\circ} \mathrm{C}$ and $510{ }^{\circ} \mathrm{C}$, respectively. In comparison, the nanocomposite samples do not exhibit the relatively sharp primary degradation step seen in the pristine polymer, instead exhibiting a more gradual decrease in weight before $225{ }^{\circ} \mathrm{C}$. One possible explanation for this is the removal of trapped solvent in the nanocomposites: DMSO, the principal solvent used in the preparation of these nanocomposites, has a boiling point of approximately $190{ }^{\circ} \mathrm{C}$, which is fairly close to the degradation temperature. However, nanocomposite samples also exhibit increased thermal stability, with the secondary and tertiary degradations occurring at $395{ }^{\circ} \mathrm{C}$ and $520{ }^{\circ} \mathrm{C}$, significant increases over pristine P3CPenT. This increased thermal stability is likely the result of the stabilizing influence of the strong chemical bonds connecting the polymer to the nanowire. 


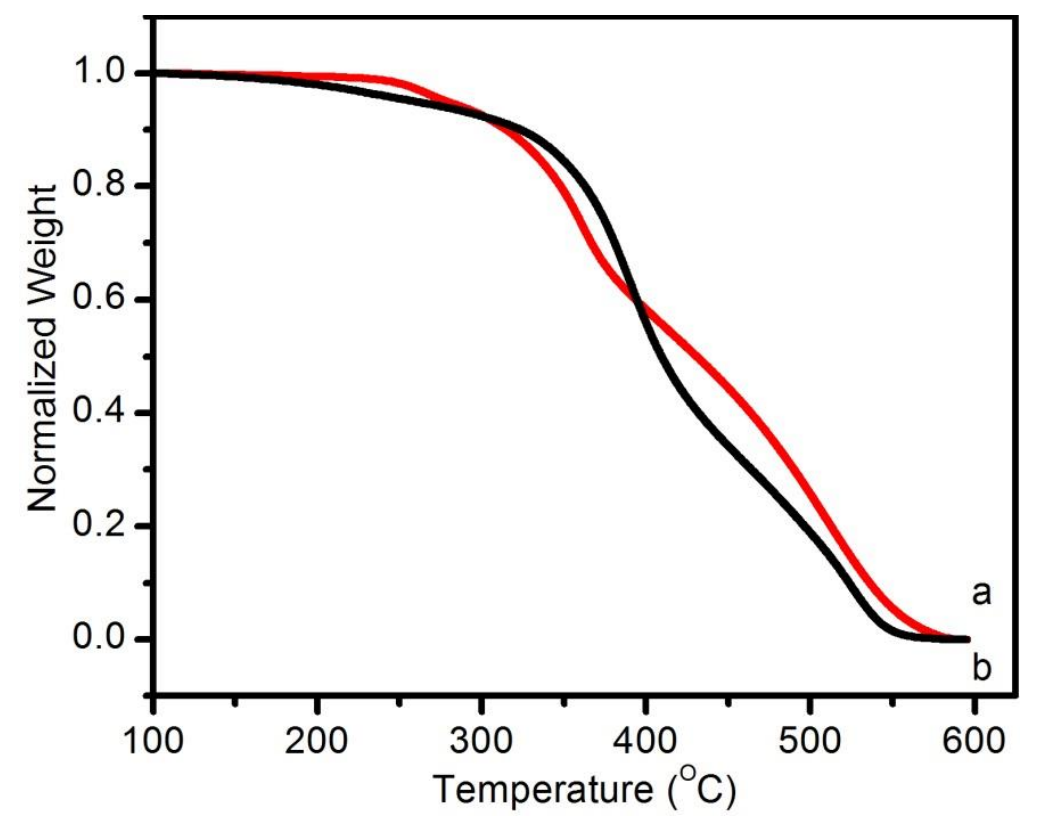

Figure 3.4: Normalized TGA heating curves for bulk pristine P3CPenT (a, red) and bulk 1:1 nanocomposite prepared in DMSO (b, black).

\subsubsection{Nanocomposite Shell: Spectral Characterization}

The UV-visible absorption spectrum of the nanocomposite (Figure 3.5) includes two major features: a peak representing the $\mathrm{ZnO}-\mathrm{NW}$ core at $\sim 370 \mathrm{~nm}$, which is in agreement with the expected band gap of $3.37 \mathrm{eV}$ $(368 \mathrm{~nm})^{35 \mathrm{a}}$, and a polymer absorption band between $400-700 \mathrm{~nm}$. Compared to the spectra of pristine P3CPenT polymer, the nanocomposite exhibits a significant red shift in the peak P3CPenT absorbance band signaling an increase in effective conjugation length ${ }^{50}$. The nanocomposite also exhibits an increase in the vibronic fine structure of the polymer, which appears with maxima at $560 \mathrm{~nm}$ and $\sim 600 \mathrm{~nm}$. This 
vibronic fine structure is attributed to $\pi-\pi$ stacking of polymer backbones in polythiophenes, commonly associated with the formation of rod conformation aggregates ${ }^{28,50-51}$. The increased fine structure of the nanocomposite indicates that the nanocomposite polymer shell exhibits a primarily rod-conformation character.

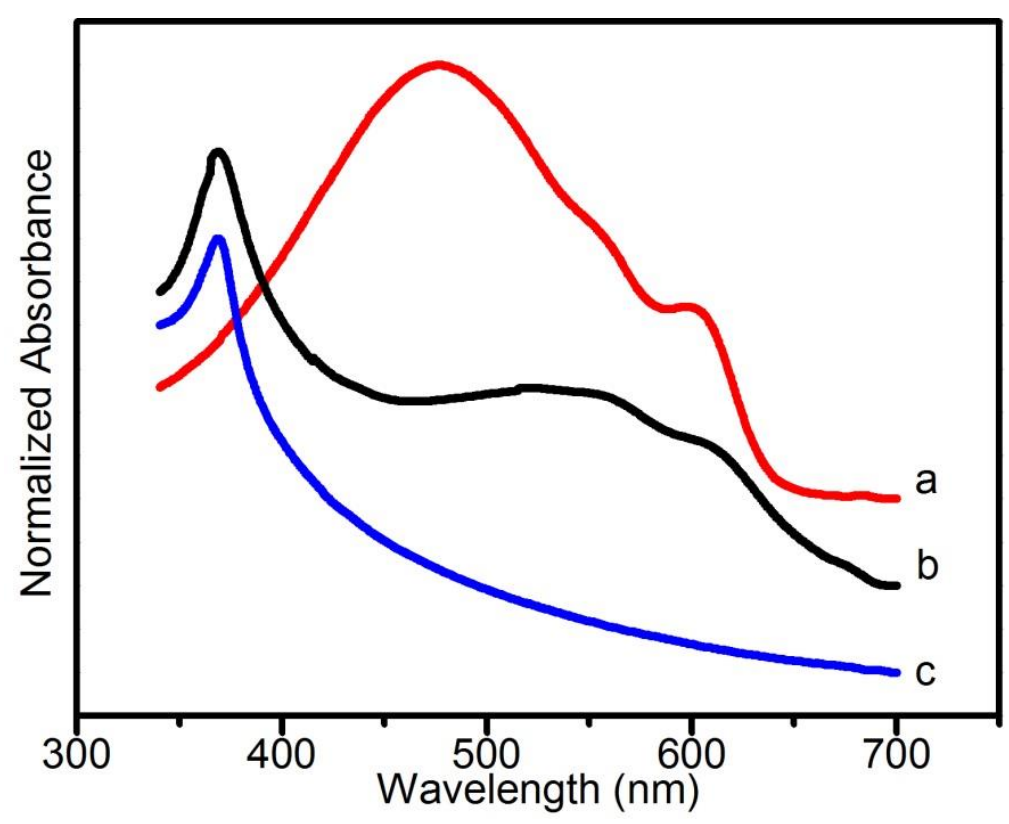

Figure 3.5: Normalized UV-vis absorption spectra of P3CPenT polymer (a), nanocomposite prepared in DMSO (b) and $\mathrm{ZnO}$ nanowires (c). All spectra shown collected from DMSO solutions (10 ug/mL).

Interestingly, the solvent in which the nanocomposite is prepared appears to have an effect on the nanocomposite polymer shell. The nanocomposite sample prepared in DMSO shows increased vibronic fine 


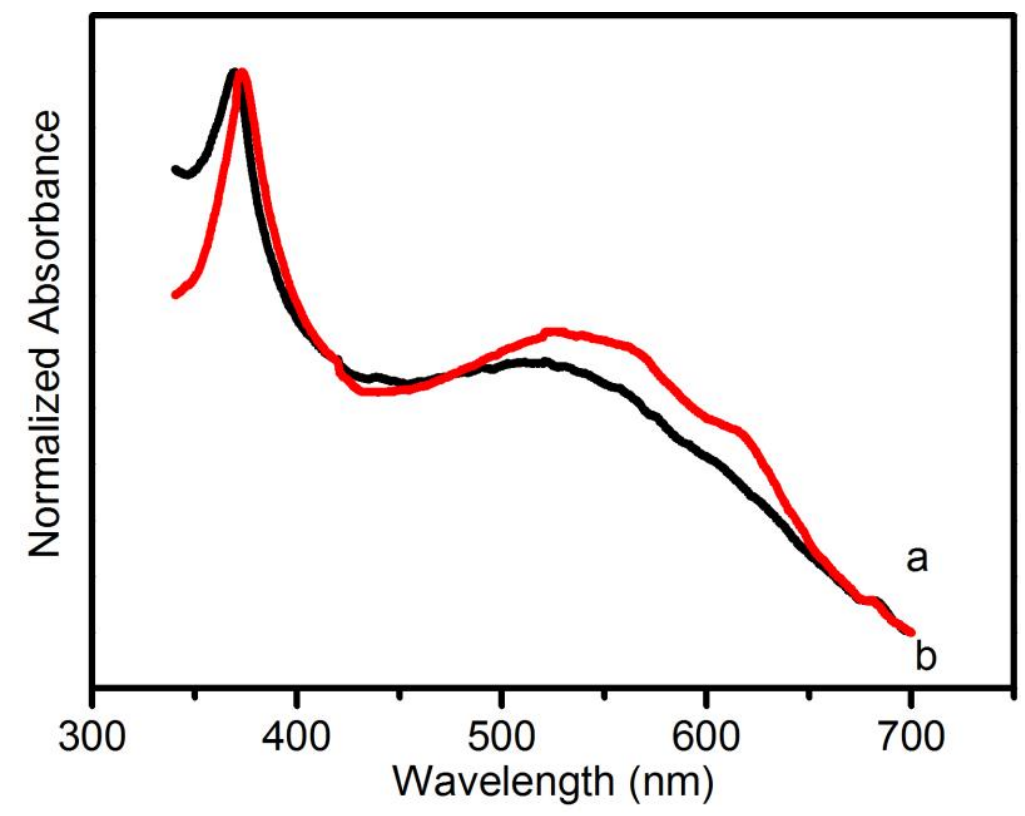

Figure 3.6: Normalized UV-Vis absorbance of nanocomposite prepared in DMSO (a, red) and pyridine (b, black) $(10 \mathrm{ug} / \mathrm{mL})$.

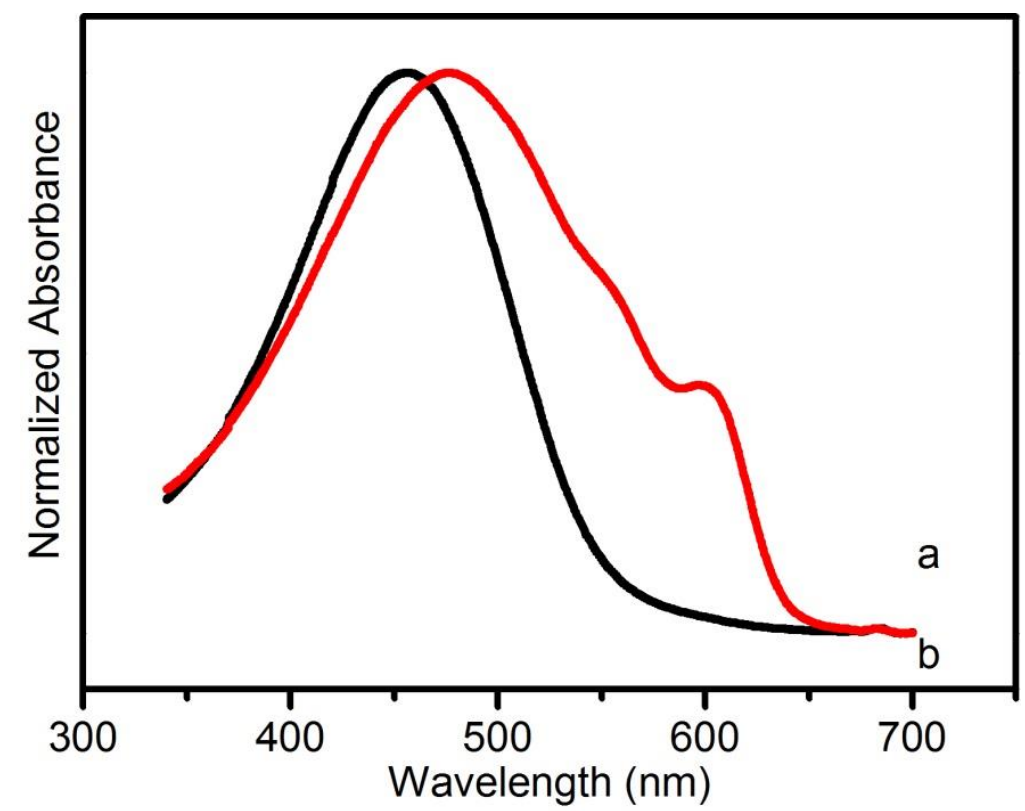

Figure 3.7: Normalized UV-Vis absorbance of P3CPenT polymer in DMSO (a, red) and pyridine (b, black) $(10 \mathrm{ug} / \mathrm{mL})$. 
structure compared to the sample prepared in pyridine (Figure 3.6), indicating increased rod character in the polymer backbone conformation. This is particularly visible in the $600 \mathrm{~nm}$ peak, which shows a significantly higher intensity in the sample prepared in DMSO compared to that of the sample prepared in pyridine. This increased rod character seen in the DMSO prepared nanocomposite may be attributed to the initial polymer conformation in solution: P3CPenT is only moderately soluble in DMSO and shows some rod character in solution (Figure 3.7 a), so the polymer shell for nanocomposite reduced DMSO exhibits increased rod character. Conversely, P3CPenT is very soluble in pyridine and shows no rod character in solution (Figure 3.7b), so the polymer shell for nanocomposites produced in pyridine may exhibit reduced rod character. This suggests that the conformation of the nanocomposite polymer shell may be influenced by solvent selection, which would enable optimization of polymer conformation in the nanocomposite through solvent selection.

The polymer shell also showed a change in crystalline structure after functionalization. The XRD pattern of the nanocomposite (Figure 3.8) is consistent with expectations, showing the wurtzite crystal structure of the $\mathrm{ZnO}^{-N^{52}}{ }^{52}$ as well as the crystalline structure of the polymer shell. However, the diffraction pattern of the nanocomposite exhibits two sharp peaks not found in the pristine polymer films at $2 \theta 5.14^{\circ}$ and $10.28^{\circ}$ representing the (100) and (200) diffraction planes of polymer side 
chain packing, respectively ${ }^{51,53}$., The peak positions indicate that the polymer shell exhibits a $d(100)$ spacing of $1.72 \mathrm{~nm}$, which is slightly greater than the reported $d(100)$ spacing for pristine P3CPenT films ${ }^{53}$. The very sharp nature of these peaks indicates a high degree of uniformity in the polymer side chain spacing along these axes, suggesting a highly crystalline polymer shell.

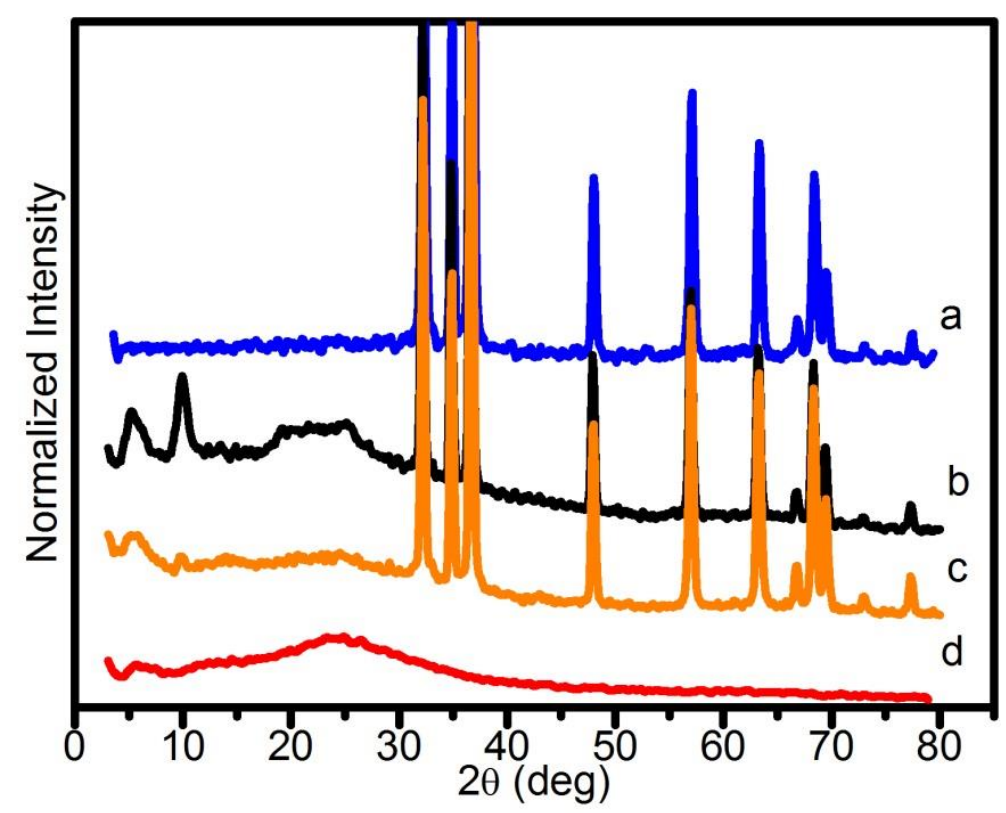

Figure 3.8: XRD patterns of $\mathrm{ZnO}$ nanowires (a), DMSO prepared nanocomposite (b), Pyridine prepared nanocomposite (c) and bulk dry P3CPenT polymer (d).

Furthermore, analysis of polymer crystal structure reveals that initial solvent again has an effect on the conformation and arrangement of the nanocomposite polymer shell. Normalized XRD patterns show that 
nanocomposite produced in DMSO exhibits markedly sharper polymer diffraction peaks compared to nanocomposite produced in pyridine ( $3.8 \mathrm{~b}$ and $3.8 \mathrm{c}$, respectively). In particular, the DMSO sample shows significantly sharper peaks at $2 \theta \sim 5^{\circ}$ and $10^{\circ}$, indicating much higher side chain regularity in the DMSO produced nanocomposite samples, suggesting higher crystallinity. The DMSO nanocomposite also shows a more narrow distribution between $2 \theta=20-30^{\circ}$ range, which is thought to be associated with backbone spacing of the polymer. This increased regularity of polymer spacing seen in the DMSO sample compared to the pyridine sample is also observed in spin coated films produced from

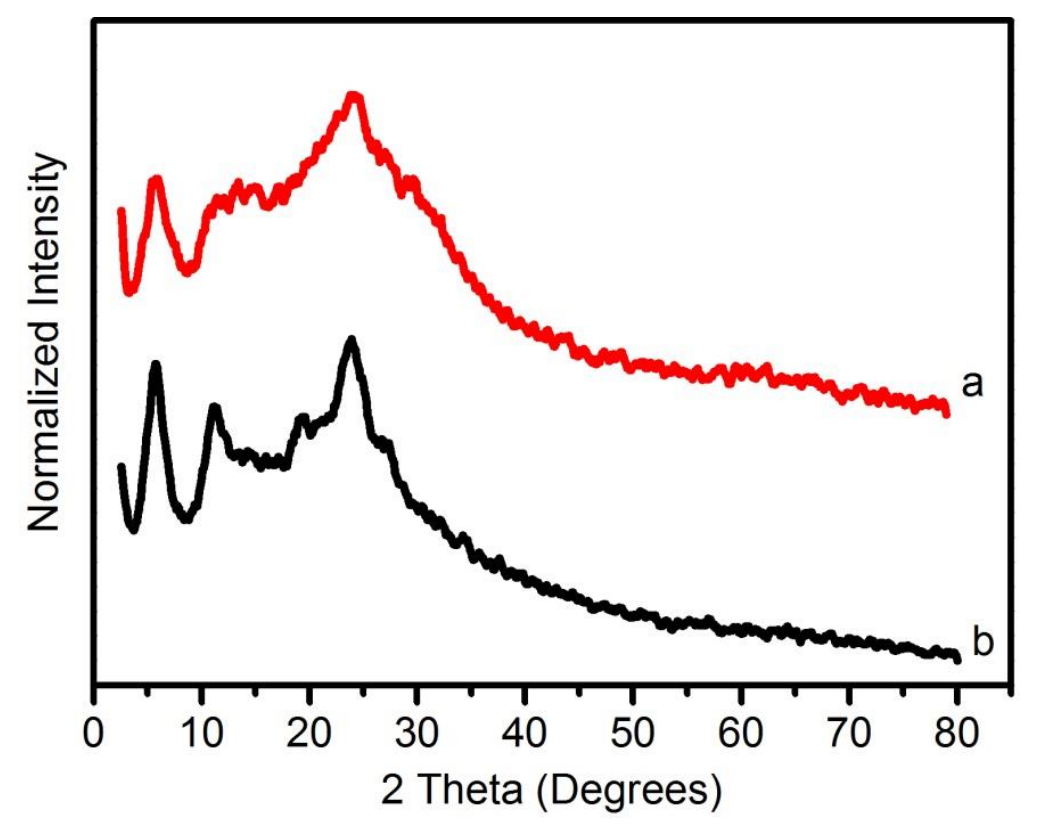

Figure 3.9: Normalized XRD patterns for spin cast P3CPenT films produced from polymer solutions in pyridine (a) and DMSO (b). 
polymer solutions (Figure 3.9), and may be explained by solvent-polymer interactions. As a poorer solvent, DMSO induces the formation of rod conformation crystalline aggregates in the polymer, as well as increasing the importance of polymer-polymer interactions in the final film organization as polymer-solvent interactions are weaker. The more consistent polymer shape and increased relative potency of polymerpolymer interactions result in decreased orientational randomness of the film. Essentially, we posit that because a higher portion of the polymer has a similar shape before being incorporated into the film, the polymer orientation in the film will be more ordered and less random.

Although conformational and orientational analysis of the nanocomposite has produced promising results, direct analysis of the performance of the nanocomposite in solar applications has not been completed due to problems in creating uniform thin films containing the nanocomposite. This essentially means that, although we have data which is suggestive of the potential of the nanocomposite, as of yet we do not have any direct measurements definitively to prove this. However, UVvisible emission spectrophotometric analysis of the samples indicates that the nanocomposite exhibits extreme quenching of polymer photoluminescent activity (Figure 3.10 ), showing nearly a $99.5 \%$ reduction in emission intensity after grafting. These results are indicative of rapid electronic transfer from the polymer shell to the nanowire core ${ }^{54}$, as the 
high energy electronic state of the polymer may be defused by transfer to the nanowire core. This is similar to the transfer of electrons from polymer shell to nanowire core which occurs during photovoltaic activity, suggesting that the nanocomposite will exhibit rapid core-shell charge transfer. Since interfacial charge transfer is one of the efficiency limiting steps in the hybrid photovoltaic process ${ }^{40}$, the highly efficient core-shell

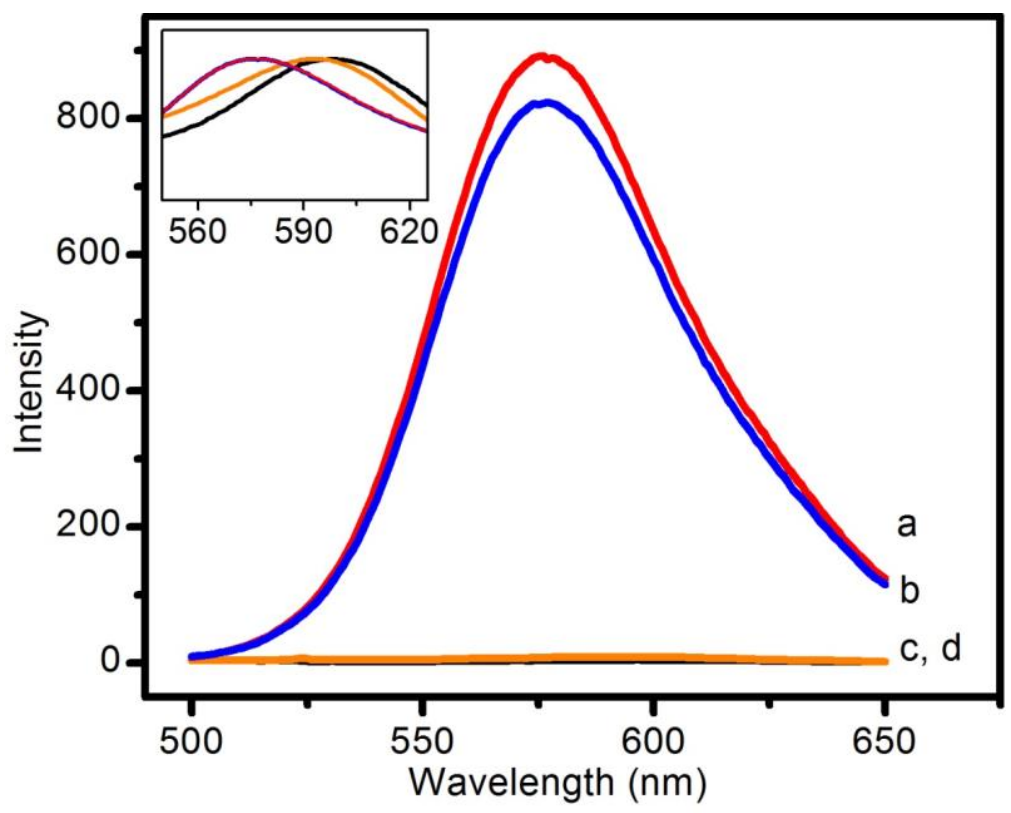

Figure 3.10: UV-visible photoluminescence spectra for pristine P3CPenT polymer (a, red), physical mixture of $\mathrm{ZnO}-\mathrm{NW}$ and pristine P3CPenT polymer ( $b$, blue), nanocomposite prepared in pyridine (c, orange) and nanocomposite prepared in DMSO (d, black). All samples measured in DMSO (12.5 ug/mL polymer). Inset displays normalized emission intensity for all samples to highlight differences in peak positions. 
charge transfer exhibited by the nanocomposite is a particularly promising sign for use in photovoltaic applications.

UV-visible emission spectra also revealed a slight shift in emission maxima after grafting, from approximately $576 \mathrm{~nm}$ in the free polymer sample to between $590-600 \mathrm{~nm}$ in the nanocomposites. This indicates a conformational extension and/or increased stacking of polymer chains in the nanocomposite, which might be attributed to the high interfacial area and energy of the nanocomposite. Additionally, the nanocomposites prepared in DMSO exhibited a slight red shift compared to those prepared in pyridine, at 598 and $593 \mathrm{~nm}$ respectively, indicative of increased conformational extension in the DMSO samples. This suggests an increased proportion of rod conformation in the DMSO samples, and is consistent with previous comparisons.

Overall, analysis of the $\mathrm{ZnO}-\mathrm{P} 3 \mathrm{CPenT}$ nanocomposites has confirmed the core-shell nature of the nanocomposite as well as the chemical nature of the core-shell linkage. Additionally, initial characterization of the polymer shell revealed increased crystalline ordering in the nanocomposite polymer shell compared to pristine P3CPenT polymer, as well as an increase in rod conformation. It was also found that solvent choice in the initial synthetic solution had an effect on the conformation of the polymer shell in the final nanocomposite product: 
nanocomposite samples produced in a poorer solvent (DMSO) showed an increase both in crystallinity and conformational extension compared to those produced in a good solvent (pyridine), suggesting that the properties of the polymer shell may be tailored to specific applications through synthetic control. Furthermore, the near complete quenching of the polymer fluorescent activity in the nanocomposite is highly suggestive of rapid core-shell electronic transfer, which is critical in photovoltaic applications. Together, these results are extremely promising for the potential of the nanocomposite in photovoltaic applications.

\subsection{Conformational Characterization of P3CATs}

Although the core-shell nanocomposite shows promise for use as the active material in photovoltaic cells, further improvements may still be made to polymer photovoltaic devices by establishing conformational and orientational control over the polymer substrates. In this vein, we have investigated the conformational response of various P3CAT polymers in solution to changes in solvation and temperature using UV-visible absorption spectrophotometry.

\subsubsection{Dilute Solution Spectrophotometry of P3CPenT Polymer}

In the case of P3CPenT polymer in a dual solvent system of $\mathrm{DMSO} / \mathrm{EtOH}$ we see that at room temperature, the polymer exhibits a distinct vibronic structure in the high ethanol content solvent systems as 
evidenced by the peaks at 550 and $600 \mathrm{~nm}$. As the DMSO concentration of the solution increases, the vibronic fine structure disappears and the spectra exhibit a blue shift (Figure 3.11). These transitions are evidence of a change in polymer conformation, with well solvated polymer adopting a coil conformation, while the poorly solvated polymer adopts a rod conformation. This conformation is well documented in $\mathrm{P}_{3} \mathrm{HT}^{28,55}$, but has yet to be investigated in great detail for carboxylated polyalkythiophenes. Systems which are relatively poor solvents for the polymer (those with high concentrations of ethanol) cause the polymer to adopt a rod conformation which has a low relative surface area of polymer exposed to the solvent. The lower conformational entropy of this state is offset by the reduced internal energy of the polymer, as the small surface area of the rod conformation minimizes the high energy unfavorable polymer-solvent interactions. As the concentration of DMSO in the system increases, the polymer-solvent interactions become more favorable, and at some point the energy of these interactions is so far reduced that they no longer compensate for the reduction in entropy of the rod conformation. When this occurs, we observe a shift in polymer conformation from coil-like to rod-like as the system minimizes free energy. 


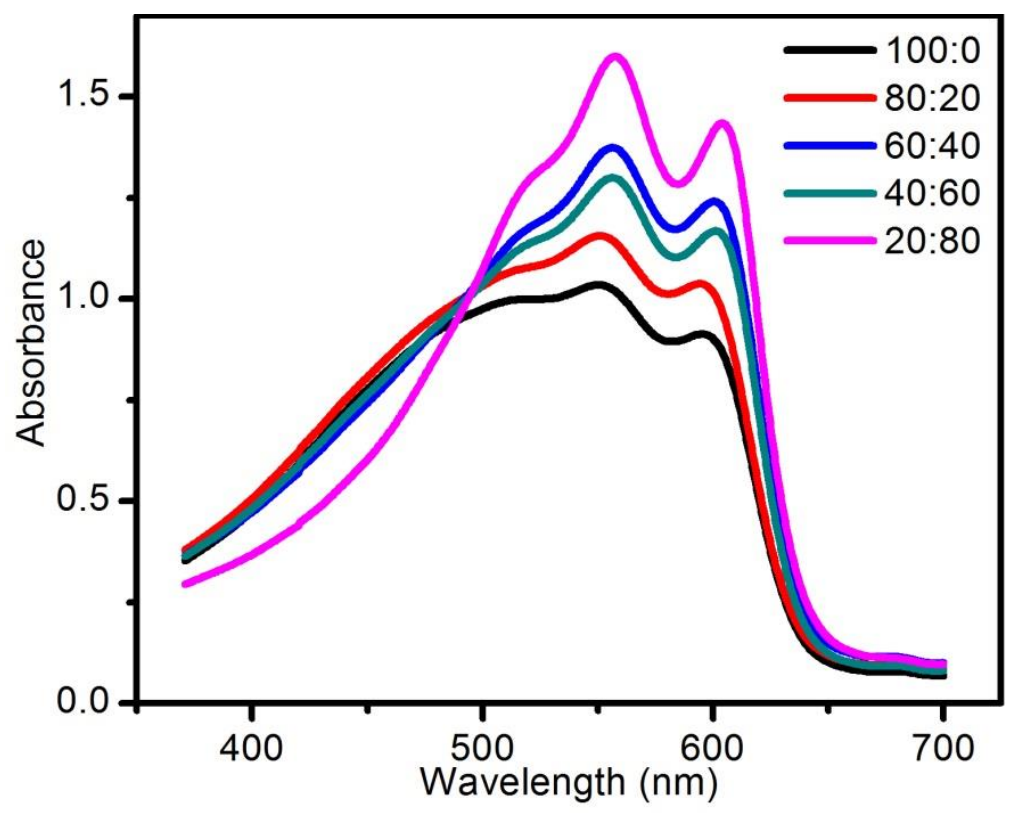

Figure 3.11: UV-Visible absorption spectra of pristine P3CPenT dissolved in solutions with varying DMSO:EtOH solvent ratios highlighting the solvatochromism of P3CPent. All spectra collected at $20^{\circ} \mathrm{C}(25 \mathrm{ug} / \mathrm{mL})$.

This explanation would lead us to expect to see a critical transitionary point in the at which the polymer quickly transitions from coil to rod as the entropic advantage of the coil conformation eclipses the lower interfacial energy of the rod conformation. Unfortunately, for the case described above, we do not see any such critical point. However, if we examine the same system at an increased temperature $\left(80^{\circ} \mathrm{C}\right)$, we do see such a point (Figure 3.12). Additionally, the higher temperature spectra generally show increased coil conformation for each individual solvent system: for example, the spectra of P3CPenT in the $60 \%$ 


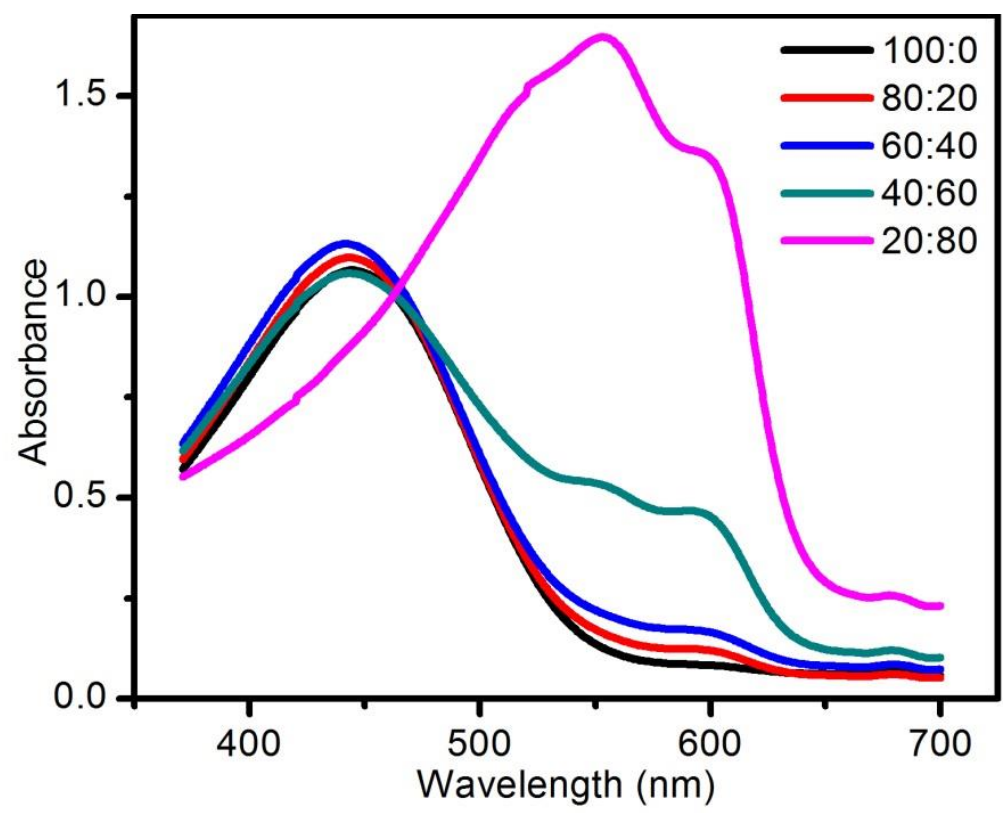

Figure 3.12: UV-Visible absorption spectra of pristine P3CPenT dissolved in solutions with varying DMSO:EtOH solvent ratios highlighting the solvatochromism of P3CPent. All spectra collected at $80^{\circ} \mathrm{C}(25 \mathrm{ug} / \mathrm{mL})$.

$\mathrm{EtOH} / 40 \%$ DMSO system shows a significant reduction in vibronic fine structure at $80{ }^{\circ} \mathrm{C}$ compared to $20{ }^{\circ} \mathrm{C}$. This is consistent with the proposed mechanism because the increased thermal energy of the polymer at higher temperatures causes the conformation to be more dominated by entropic constraints, thus adopting the more entropically favorable coiled conformation. Furthermore, this suggests that the effects of temperature on polymer are similar in effect and mechanism to the effects of solvation.

In order to further confirm this theory, the effects of temperature on the conformation of P3CPenT polymers was also characterized using UV- 
visible spectrophotometry. The polymer was tested in an ideal solvent system $(80 \% \mathrm{DMSO} / 20 \% \mathrm{EtOH}$, as determined by the previous solvent effects testing) in order to magnify the effects of changes in temperature on polymer conformation. As can be seen in the UV-visible spectra (Figure 3.13), the polymer absorbance spectrum exhibits a red shift as the temperature of the solution is increased. Furthermore, the fine vibronic structure of the polymer denoted by peaks at approximately 550 and 600 $\mathrm{nm}$, largely disappears as the temperature increases past a critical value, suggesting a polymer conformational transfer from coil to rod.

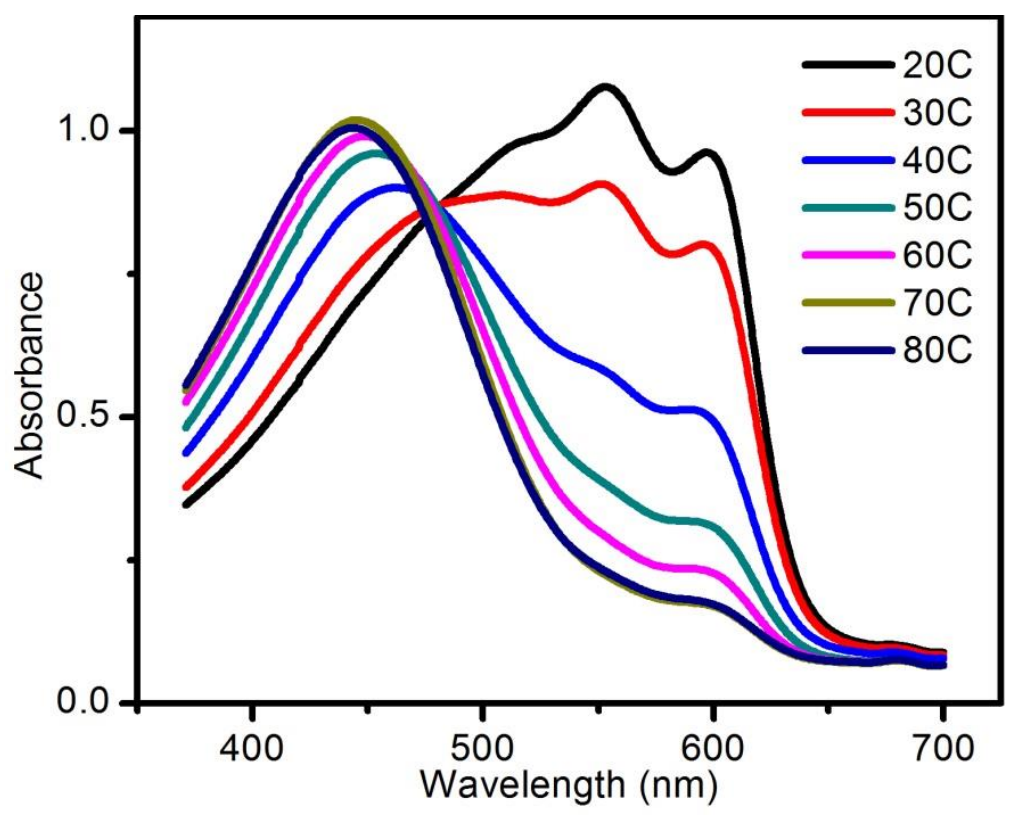

Figure 3.13: UV-Visible absorption spectra of pristine P3CPenT polymer in a solution of $80 \%$ DMSO: $20 \%$ EtOH at varying temperatures $(25 \mathrm{ug} / \mathrm{mL})$. 
This thermochromism is very similar to the solvochromism observed in the solvent testing, providing further evidence that both the thermochromism and solvatochromism are the result of conformational changes driven by the differential between the entropic favorability of the coil conformation and the reduced high energy interactions of the rod conformation. At lower temperatures, the entropic advantages of the coil conformation are not strong enough to overcome the relatively poor polymer-solvent interactions, causing the chains to organize into the lower surface volume rod conformation. However, as temperatures rise the influence of entropy on free energy of the system increases and the entropically favored coil conformation becomes the dominant polymer conformation, causing the polymer solution to change color.

Similarly to solvatochromism, we expect to see a critical transitional temperature at which the conformation of the polymer changes rapidly. UV-visible heating curves indicate that this critical temperature occurs at approximately $30{ }^{\circ} \mathrm{C}$ for $\mathrm{P} 3 \mathrm{CPenT}$ in the solvent system tested (Figure $3.14,90: 10)$, although obviously this temperature is heavily dependent upon a number of factors such as solution composition, the temperature at which the polymer was equilibrated at and the heating rate. Furthermore, although the thermochromism appears to be reversible, the results suggest the presence of significant rate-dependant hysteresis, with more rapid temperature changes causing greater hysteresis. 


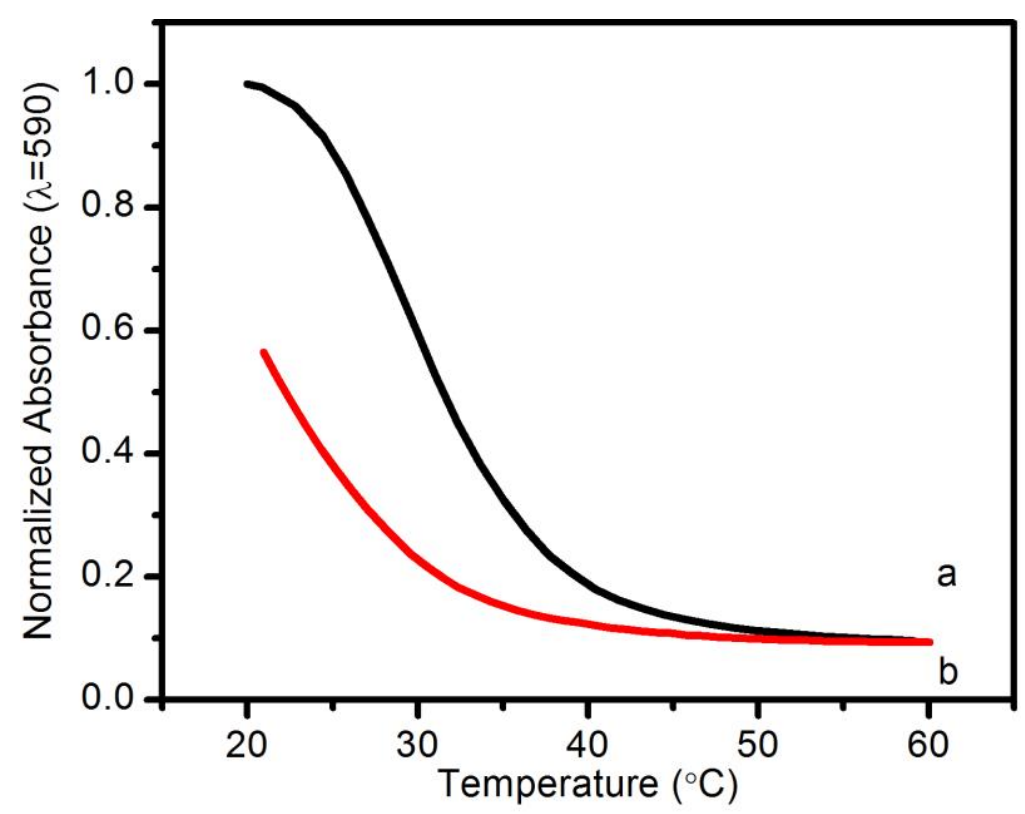

Figure 3.14: UV-Visible absorption heating curve (a) and cooling curve (b) for P3CPenT in 80\% DMSO: 20\% EtOH $(25 \mathrm{ug} / \mathrm{mL})$.

\subsubsection{Effect of Side-Chain Length on Conformation}

The effects of alkyl chain side length on the conformational transition of P3CAT polymers were also characterized. As may be seen in the UV-visible spectra (Figure 3.15), polymer side chain length appears to have a significant effect on polymer conformation. Polymers with longer side chains exhibit greater fine structure peaks compared to the shorter side chain polymers, which would suggest that polymers with longer side chains greater have a greater propensity to adopt a rod conformations in the solvent system tested. 


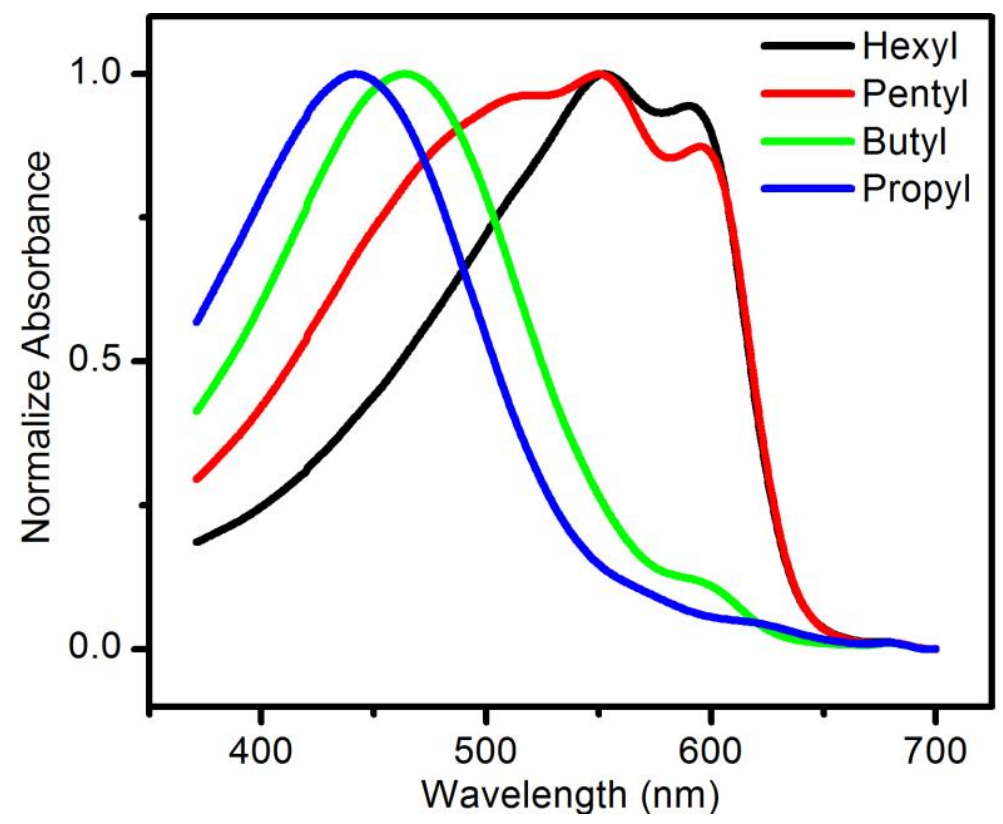

Figure 3.15: UV-visible absorption spectra of P3CAT polymers at 20C in 100\% DMSO:0\% EtOH solution (25 ug/mL).

Indeed, further investigation of the effects of side chain length on polymer conformational transitions yielded data consistent with this trend. Characterization of the solvatochromism of the polymer conformational transition in a DMSO:EtOH solvent system revealed that, as the length of the side chain increased, the concentration of ethanol required to induce rod conformation decreased, showing a greater propensity to adopt rod conformation (Figure 3.16). These results are likely due to changes in the polymer solvent interactions: changing the length of the nonpolar alkyl sidechains should cause a decrease in the overall solubility of the molecule in polar and moderately polar solvents. The decrease in 

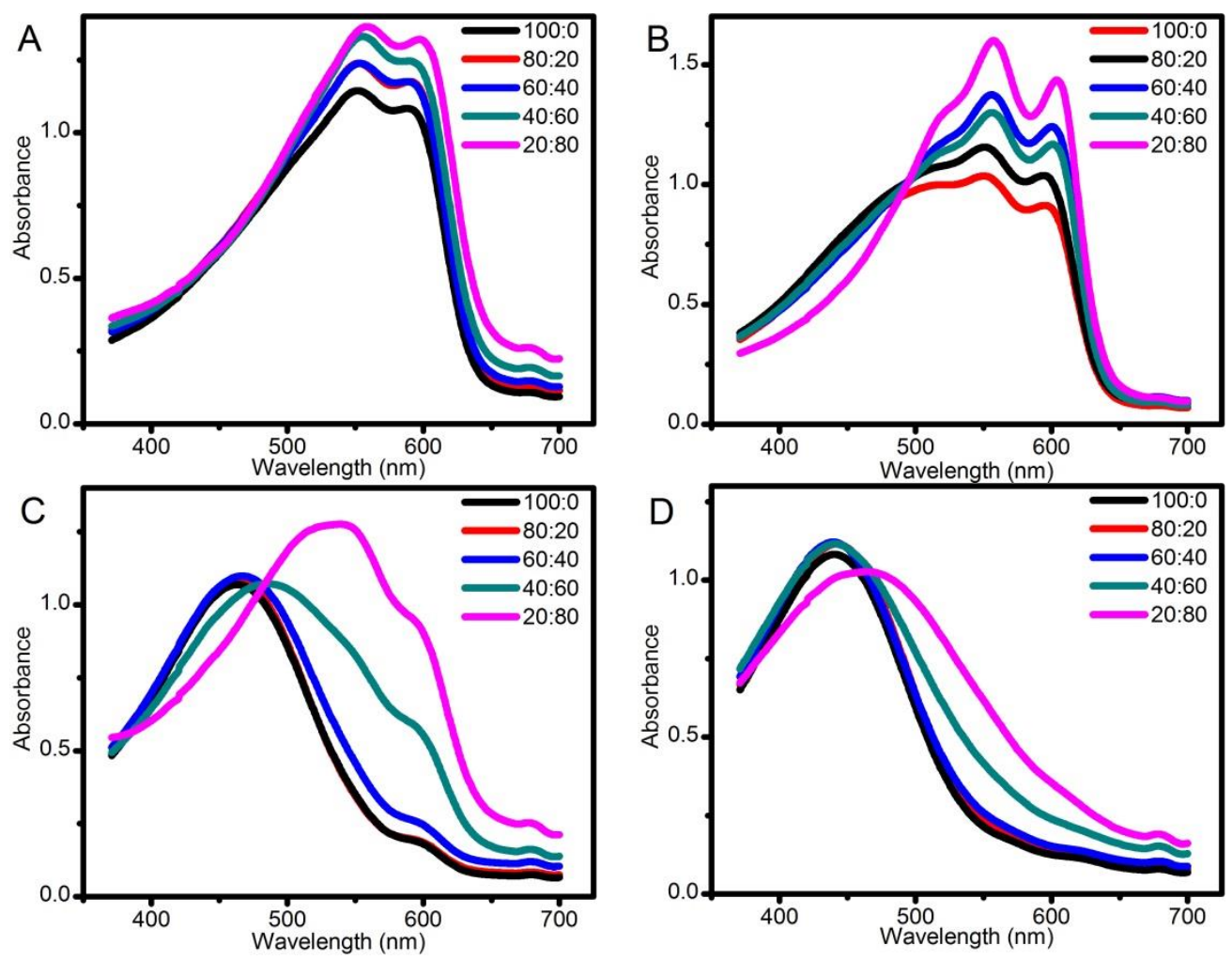

3.16: UV-Visible absorption spectra of P3CHexylT (A), P3CPenT (B), P3CButylT (C) and P3CPropylT (D) in solutions of varying DMSO:EtOH solvent ratios at $20^{\circ} \mathrm{C}(25 \mathrm{ug} / \mathrm{mL})$.

solubility essentially means that interactions between the polymer molecule and the solvent are increased in energy, thus causing the high surface area coil conformation to be less energetically favorable, as it increases the overall free energy of the system. This is consistent with our investigations of P3CPenT polymer: the more unfavorable the solventpolymer interactions, the more rod conformation exhibited by the polymer in solution. 


\subsubsection{Kinetics of Conformational Transition in P3CPenT}

In order to further understand the mechanism of the coil-to-rod transition of the solvated polymer, UV-visible absorption spectrophotometry was used to study the time-dependent chromism of P3CPenT polymer, in the hopes of being able to investigate the rate of the conformational transition. Unfortunately, the initial study proved disappointing, as the polymer showed no spontaneous time dependent chromism. However, we were able to thermally induce the conformational transition by subjecting the polymer sample to rapid temperature changes

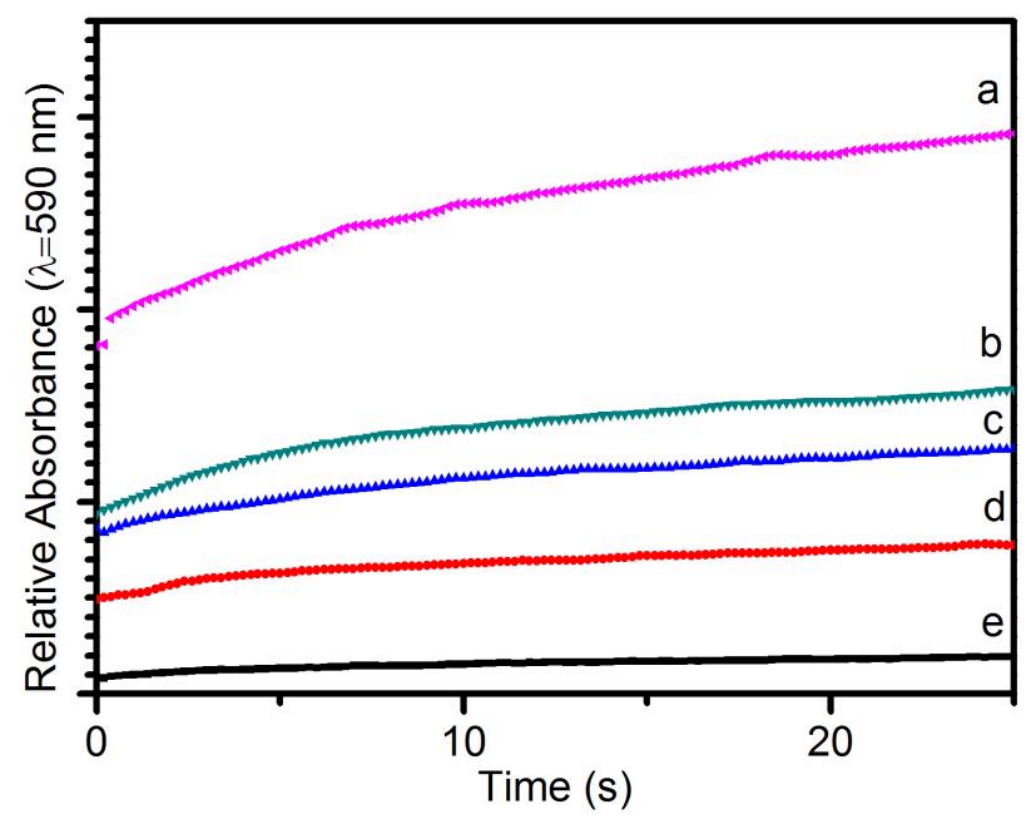

Figure 3.17: Change in absorbance $(\lambda=590)$ with respect to time for 40 ug/mL P3CPenT (a), 25 ug/mL P3CPenT (b), 15 ug/mL P3CPenT (c), 10 $\mathrm{ug} / \mathrm{mL}$ P3CPenT (d) and $5 \mathrm{ug} / \mathrm{mL}$ P3CPenT (e). 
in order to study the time dependent chromism (Figure 3.17). It is worth noting that the thermally induced nature of these conformational transitions results in extremely rapid conformational changes (where initial rates are determined over a time scale of several seconds), which may contribute some inaccuracy to the resulting initial rates.

The polymer exhibits what appears to be a first order rate law. In theory, the rate is predicted to scale with concentration by the equation Rate $\propto[\text { Polymer }]^{n}$, in which the exponent determines the reaction order. In the case of $\mathrm{P} 3 \mathrm{CPenT}$ in the solvent tested, the data points may be modeled by the proportional relationship Rate $\propto[P]^{0.93 \pm 0.12}$

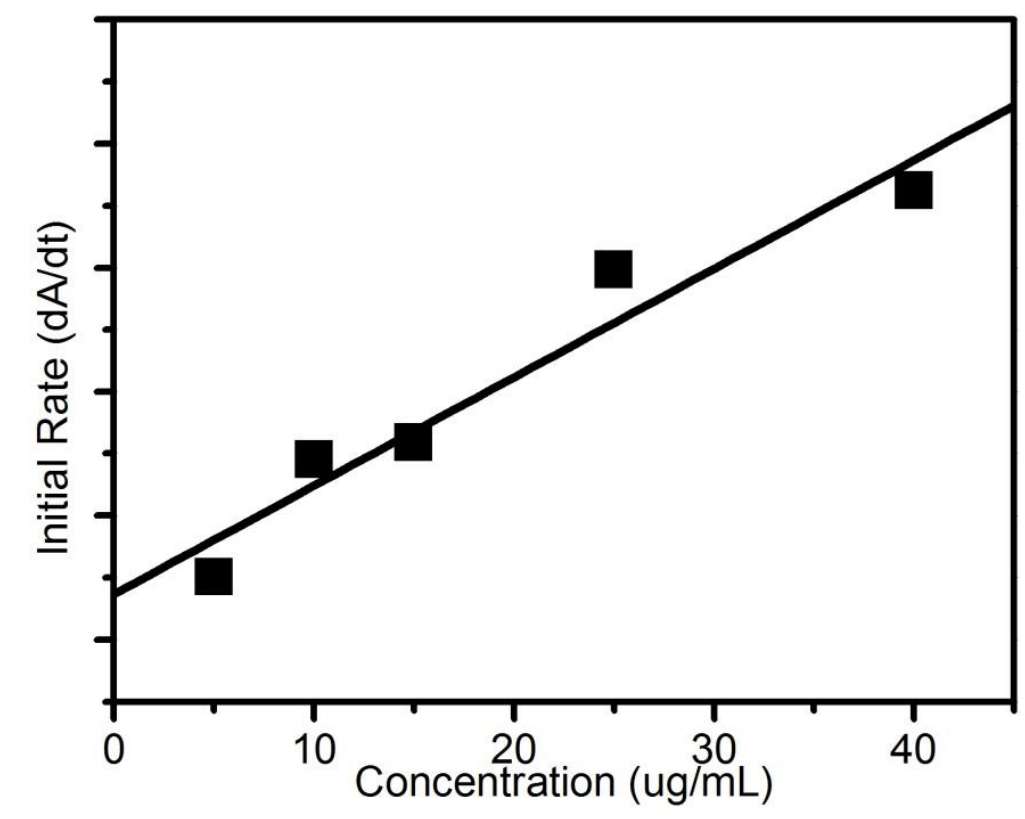

Figure 13.18: Initial rate plot fitted with a power function (black line) determined from the kinetic data for P3CPenT shown in Figure 13.17. 
(Figure 13.18).This first order relationship implies a linear, proportional increase in rate as concentration increases. This is consistent with established literature results for the conformational coil-to-rod transition of P3HT in a marginal organic solvent, and is what we would expect for a conformational change, which may essentially be thought of as a single reactant reaction.

Interestingly, a newer batch of P3CPenT polymer has shown time dependent chromism (Figure 13.19). This is troubling, because it implies that there may be some differences between the two batches of polymers which could be problematic for further studies involving P3CPenT.

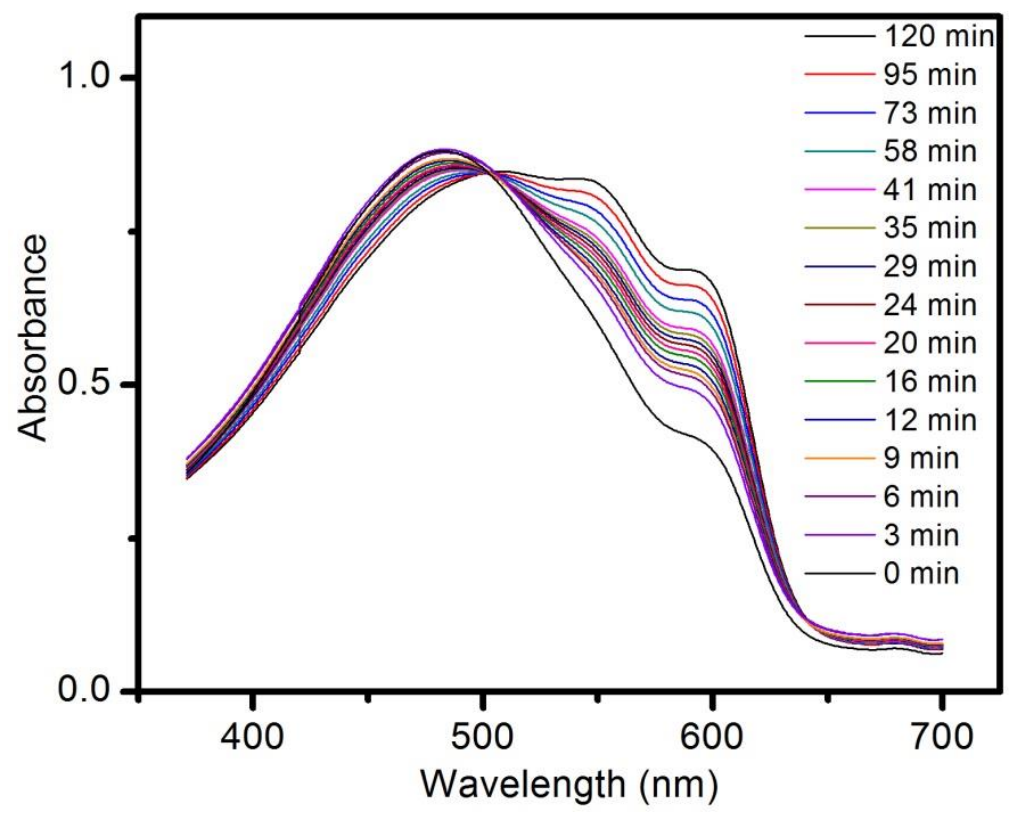

Figure 13.19: Time dependent chromism of new P3CPenT samples in 100\% DMSO:0\% EtOH solution (25 ug/mL). 
However, it also allows for the study of time dependent chromism without the use of thermally induced transitions, which should make it possible to collect more accurate data to confirm our results. Unfortunately, there was not time for this to be completed before the project ended, but this still presents an opportunity for future studies in our group to confirm our work with this polymer. 


\section{3: Research Outlook}

Although our initial characterization of the nanocomposite has yielded some promising results, in order to truly confirm its potential as a photovoltaic substrate material we need to successfully incorporate it into a solar cell. Once we've done this, directly testing the efficiency and comparing it to a cell which does not contain nanocomposite may give us a clear picture of how much of an improvement the nanocomposite represents. To this effect, our lab is in the process of trying to create a working solar cell based on nanocomposite.

Further examination of the core-shell nanocomposite based on carboxylated polyalkylthiophenes is also in order. Although we have done a great deal of characterization of the P3CPenT/ZnO nanocomposite produced from DMSO and pyridine, there may be other solvents which can produce even more ordered polymer shells. Additionally, further study is warranted to determine the effects of polymer side-chain length on the loading and conformation of the core-shell nanocomposite. Samples of nanocomposite have been produced using a propyl side-chain P3CAT, and yielded an orange colored product (Figure 3.20), but further investigation of these different side-chain nanocomposite is required. The general strategy of employing side-chain functionalized CPs to create core-shell nanocomposites may also be extended to other nanoparticles 
such as quantum dots or $\mathrm{TiO}_{2}$ nanorods in the future. This could be particularly interesting in the case of quantum dots, which have attracted great interest in recent years due to their good optoelectronic properties.

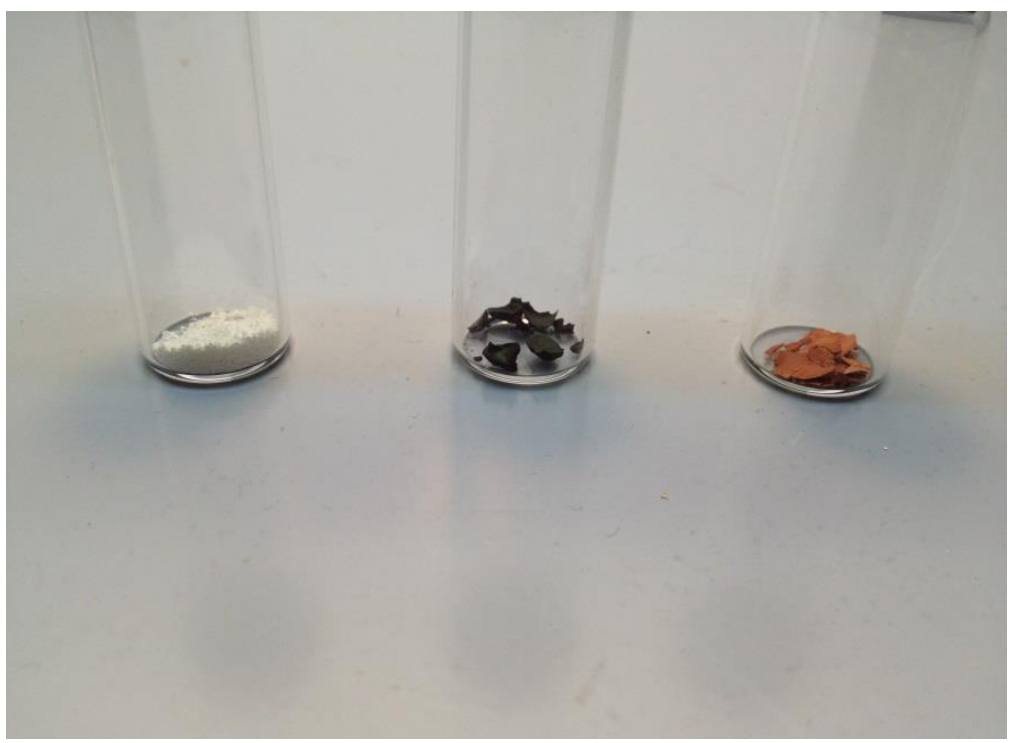

Figure 3.20: Visual picture of ZnO-NW (left), P3CPenT nanocomposite (center) and P3CProT nanocomposite (right).

Another area of future work for this project is the study of higher concentration liquid crystal (LC) solutions. At higher concentrations, polymer solutions form a LC phase exhibiting birefringence. Although this is an area which has received some study in $\mathrm{P} 3 \mathrm{HT}$, little work has been done in this area with P3CPenT. Initial investigation of high concentration P3CPenT films containing suspended nanocomposites were promising as the films exhibited strong birefringence (Figure 3.21), but further characterization of this phenomenon is in order. This system is 
particularly interesting, as the coil-to-rod transition of P3CPenT may also have considerable effects on the development of the LC phase. Based on the Onsager hard-rod model, the emergence of the LC phase is due to a tendency of cylindrical objects to coaxially orient, as a result of increased positional entropy. P3CPenT's conformational duality may provide another mechanism to control lyotropic LC ordering, which would be highly desirable for large scale device production.

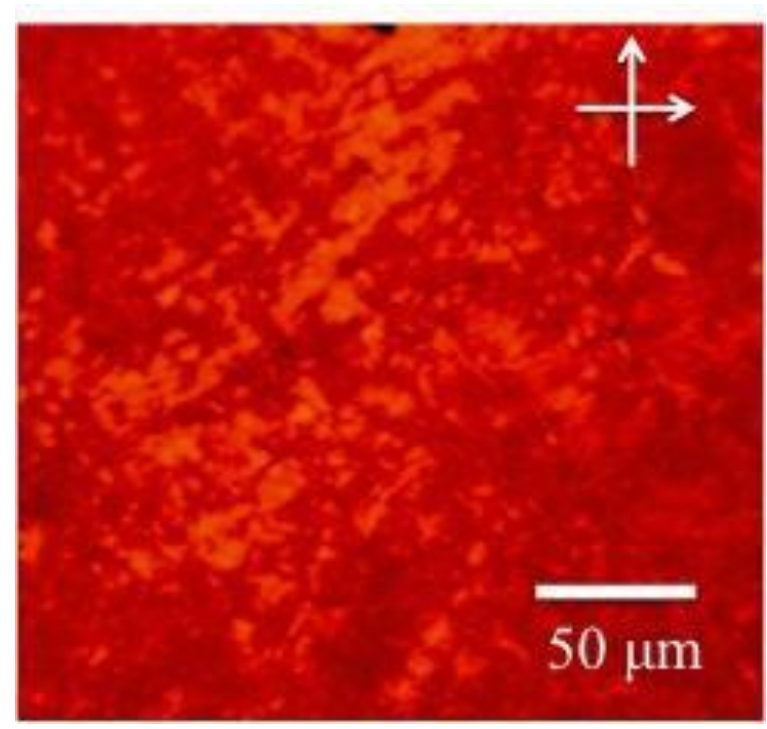

Figure 3.21: Optical imaging of the core-shell nanocomposites under cross polars (represented by the arrows) suggests the formation of a lyotropic liquid crystalline phase, as indicated by the strong birefringence. 


\section{Conclusions}

In summary, the kinetics and thermodynamics of the rod-coil conformational transition of P3CAT polymers have been investigated. This conformational chromism may be initiated by a number of factors including temperature and solvent changes, and the phase transition exhibits a first order rate law. The chromism is also affected by the structure of the polymer, with more nonpolar polymers adopting the rod conformation more readily in the solvent system tested. Control over this conformational transition is critical for the improvement of polythiophene based electronic devices, as conformation plays a large role in determining the conductivity of conducting polymers.

Additionally, an anisotropic core-shell hybrid nanocomposite was synthesized by covalently grafting the carboxylic acid side-functionalized $\mathrm{P} 3 \mathrm{HT}$ known as P3CPenT onto semiconducting $\mathrm{ZnO}$ nanowires. The P3CPenT shell of the nanocomposite exhibits improved crystallinity as compared to the pristine polymer in bulk, and the morphology of the polymer shell appears to be influenced by the morphology of the polymer in the reaction medium. The nanocomposite polymer shell also exhibits elongated conjugation length of the polymer backbone and rapid charge transfer at the polymer-nanowire interface, which suggests the potential for increased performance in electronic applications. 
These achievements address the critical issue in CP solar devices of improving device performance. Conformational control over the active polymer layer is crucial for the development of polymer films with optimized electronic properties, which are likewise crucial for the success of polymer photovoltaics. Likewise, the direct side-on grafting achieved in the novel core-shell nanocomposite represents a breakthrough in inorganic nanoparticler/CP hybrid nanocomposites. Together, this work represents a foundation for the construction of $\mathrm{CP}$ solar devices with highly ordered polymer structure and rapid charge carrier transport. 


\section{References}

1. Tsao, J.; Lewis, N.; Crabtree, G., Solar FAQs. Energy, U. S. D. o., Ed. Sandia National Labs, 2006; p 24.

2. Williams, R. Journal of Chemical Physics, 1960, 32 (5), 1505-1514.

3. Fraas, L. M.; Partain, L. D., Solar Cells and Their Applications. 2 ed.; John Wiley \& Sons, Inc.: 2010; p 627.

4. Chapin, D.; Fuller, C.; Pearson, G. Journal of Applied Physics, $1954,25,676-677$.

5. Roston, E., Solar Silicon Price Drop Brings Renewable Power Closer. Bloomberg New Energy Finance 2012.

6. Shirakawa, H.; Louis, E. J.; Macdiarmid, A. G.; Chiang, C. K.; Heeger, A. J. Journal of the Chemical Society, Chemical Communications, 1977, 132, 578-580.

7. Burroughes, J. H.; Bradley, D. D. C.; Brown, A. R.; Marks, R. N.; Mackay, K.; Friend, R. H.; Burns, P. L.; Holmes, A. B. Nature, 1990, 347 (6293), 539-541.

8. Ye, Q.; Chi, C., Conjugated Polymers for Organic Solar Cells, Solar Cells - New Aspects and Solutions. In Solar Cells - New Aspects and Solutions, Kosyachenko, L. A., Ed. InTech: 2011.

9. Søndergaard, R.; Hösel, M.; Angmo, D.; Larsen-Olsen, T. T.; Krebs, F. C. Materials Today, 2012, 15 (1-2), 36-49. 
10. Green, M. A.; Emery, K.; Hishikawa, Y.; Warta, W.; Dunlop, E. D. Progress in Photovoltaics: Research and Applications, 2013, 21 (5), 827-837.

11. NREL, Best Research-Cell Efficiencies. Energy, D. o., Ed. 2013.

12. Facchetti, A. Materials Today, 2013, 16 (4), 123-132.

13. Jørgensen, M.; Norrman, K.; Gevorgyan, S. A.; Tromholt, T.; Andreasen, B.; Krebs, F. C. Advanced Materials, 2012, 24 (5), 580612.

14. (a) Tsuchihara, K.; Masuda, T.; Higashimura, T. Journal of Polymer Science Part A: Polymer Chemistry, 1991, 29 (4), 471-478; (b) Chambon, S.; Rivaton, A.; Gardette, J.-L.; Firon, M. Solar Energy Materials and Solar Cells, 2007, 91 (5), 394-398; (c) Chambon, S.; Rivaton, A.; Gardette, J.-L.; Firon, M.; Lutsen, L. Journal of Polymer Science Part A: Polymer Chemistry, 2007, 45 (2), 317-331; (d) Abdou, M. S. A.; Orfino, F. P.; Son, Y.; Holdcroft, S. Journal of the American Chemical Society, 1997, 119 (19), 4518-4524.

15. (a) So, F.; Kondakov, D. Advanced Materials, 2010, 22 (34), 37623777; (b) Rabek, J. F., Polymer Photodegradation: Mechanisms and Experimental Methods. Chapman \& Hall: 1995.

16. Beek, W. J. E.; Wienk, M. M.; Kemerink, M.; Yang, X.; Janssen, R. A. J. The Journal of Physical Chemistry B, 2005, 109 (19), 95059516. 
17. (a) Henry, B. M.; Dinelli, F.; Zhao, K. Y.; Grovenor, C. R. M.; Kolosov, O. V.; Briggs, G. A. D.; Roberts, A. P.; Kumar, R. S.; Howson, R. P. Thin Solid Films, 1999, 355-356 (0), 500-505; (b) Chawdhury, N.; Köhler, A.; Harrison, M. G.; Hwang, D. H.; Holmes, A. B.; Friend, R. H. Synthetic Metals, 1999, 102 (1-3), 871-872.

18. Wang, M.; Xie, F.; Xie, W.; Zheng, S.; Ke, N.; Chen, J.; Zhao, N.; Xu, J. B. Applied Physics Letters, 2011, 98 (18), - .

19. Saritas, M.; McKell, H. D. Solid-State Electronics, 1988, 31 (5), 835-842.

20. Mikhnenko, O. V.; Azimi, H.; Scharber, M.; Morana, M.; Blom, P. W. M.; Loi, M. A. Energy \& Environmental Science, 2012, 5 (5), 69606965.

21. Li, Y.; Zou, Y. Advanced Materials, 2008, 20 (15), 2952-2958.

22. (a) Deibel, C.; Wagenpfahl, A.; Dyakonov, V. physica status solidi (RRL) - Rapid Research Letters, 2008, 2 (4), 175-177; (b) Arkhipov, V. I.; Heremans, P.; Emelianova, E. V.; Adriaenssens, G. J.; Bässler, H. Applied Physics Letters, 2003, 82 (19), 3245-3247.

23. Roth, S.; Carroll, D., One-Dimensional Metals. Wiley: 2004.

24. Geoghegan, M.; Hadziioannou, G., Polymer Electronics. OUP Oxford: 2013.

25. (a) Yang, X.; Loos, J.; Veenstra, S. C.; Verhees, W. J. H.; Wienk, M. M.; Kroon, J. M.; Michels, M. A. J.; Janssen, R. A. J. Nano 
Letters, 2005, 5 (4), 579-583; (b) Chen, F.-C.; Tseng, H.-C.; Ko, C.J. Applied Physics Letters, 2008, 92 (10), -; (c) Geng, J.; Zeng, T. Journal of the American Chemical Society, 2006, 128 (51), 1682716833.

26. (a) Wu, Y.; Li, Z.; Ma, W.; Huang, Y.; Huo, L.; Guo, X.; Zhang, M.; Ade, H.; Hou, J. Advanced Materials, 2013, 25 (25), 3449-3455; (b) Kim, J. S.; Lee, J. H.; Park, J. H.; Shim, C.; Sim, M.; Cho, K. Advanced Functional Materials, 2011, 21 (3), 480-486.

27. Xu, W.; Li, L.; Tang, H.; Li, H.; Zhao, X.; Yang, X. The Journal of Physical Chemistry B, 2011, 115 (20), 6412-6420.

28. Rughooputh, S. D. D. V.; Hotta, S.; Heeger, A. J.; Wudl, F. J. Journal of Polymer Science Part B: Polymer Physics, 1987, 25, 10711078.

29. Rodd, C. M.; Agarwal, R. Nano Letters, 2011, 11, 3460-3467.

30. Ma, W.; Yah, W. O.; Otsuka, H.; Takahara, A. Beilstein Journal of Nanotechnology, 2012, 3, 82-100.

31. (a) Li, L.; Li, B.; Hood, M. A.; Li, C. Y. Polymer, 2009, 50 (4), 953965; (b) Grell, M.; Bradley, D. D. C.; Inbasekaran, M.; Woo, E. P. Advanced Materials, 1997, 9 (10), 798-802; (c) Lim, E.; Jung, B.-J.; Lee, J.; Shim, H.-K.; Lee, J.-I.; Yang, Y. S.; Do, L.-M. Macromolecules, 2005, 38 (10), 4531-4535. 
32. (a) Li, L.; Lu, G.; Yang, X. Journal of Materials Chemistry, 2008, 18 (17), 1984-1990; (b) Bertho, S.; Janssen, G.; Cleij, T. J.; Conings, B.; Moons, W.; Gadisa, A.; D’Haen, J.; Goovaerts, E.; Lutsen, L.; Manca, J.; Vanderzande, D. Solar Energy Materials and Solar Cells, 2008, 92 (7), 753-760.

33. Lin, Z. Chemistry - A European Journal, 2008, 14 (21), 6294-6301.

34. Milliron, D. J.; Alivisatos, A. P.; Pitois, C.; Edder, C.; Fréchet, J. M. J. Advanced Materials, 2003, 15 (1), 58-61.

35. (a) Zhang, S. J.; Majewski, P. W.; Keskar, G.; Pfefferle, L. D.; Osuji, C. O. Langmuir, 2011, 27, 11616-11621; (b) Zhang, S. J.; Pelligra, C. I.; Keskar, G.; Jiang, J.; Majewski, P. W.; Taylor, A. W.; IsmailBeigi, S.; Pfefferle, L. D.; Osuji, C. O. Advanced Materials, 2012, 24, 82-87.

36. (a) Huynh, W. U. D., Janke, J.; Alivisatos, Paul. Science, 2002, 295, 2425-2427; (b) Locklin, J.; Patton, D.; Deng, S.; Baba, A.; Millan, M.; Advincula, R. C. Chemistry of Materials, 2004, 16, 51875193.

37. (a) Qiao, Q.; Su, L.; Beck, J.; McLeskey, J. T. Journal of Applied Physics, 2005, 98 (9), -; (b) Xu, T.; Yan, M.; Hoefelmeyer, J. D.; Qiao, Q. Royal Society of Chemistry Advances, 2012, 2, 854-862. 
38. (a) Ren, S.; Bernardi, M.; Lunt, R. R.; Bulovic, V.; Grossman, J. C.; cak, S. G. Nano Letters, 2011, 11, 5316-5321; (b) Zhao, L.; Li, Y.; Liu, Z.; Shimizu, H. Chemistry of Materials, 2010, 22, 5949-5956.

39. (a) Lin, Y. Y.; Lee, Y. Y.; Chang, L.; Wu, J. J.; Chen, C. W. Applied Physics Letters, 2009, 94, 063308; (b) Briseno, A. L.; Holcombe, T. W.; Boukai, A. I.; Garnett, E. C.; Shelton, S. W.; Frechett, J. W. J.; Yang, P. Nano Letters, 2010, 10, 334-340.

40. Zhao, L.; Wang, J.; Lin, Z. Frontiers of Chemistry in China, 2010, 1, 33-34.

41. Nanotechnology and Food Safety. Department, F. a. E. H., Ed. Hong Kong, China, 2010.

42. Arici, E.; Meissner, D.; Shaffler, F.; Sariciftci, N. S. International Journal of Photoenergy, 2003, 5, 199-208.

43. (a) Zhang, Q.; Russell, T. P.; Emrick, T. Chemistry of Materials, 2007, 19 (15), 3712-3716; (b) Lin, Y. H.; Jiang, C.; Xu, J.; Lin, Z.;

Tsukruk, V. V. Advanced Materials, 2007, 19 (22), 3827-3832; (c) Querner, C.; Benedetto, A.; Demadrille, R.; Rannou, P.; Reiss, P. Chemistry of Materials, 2006, 18 (20), 4817-4826.

44. Fukushima, T.; Aida, T. Chemistry - A European Journal, 2007, 13 (18), 5048-5058. 
45. Liu, J.; Tanaka, T.; Sivula, K.; Alivisatos, A. P.; Fréchet, J. M. J. Journal of the American Chemical Society, 2004, 126 (21), 65506551.

46. Worfolk, B. J.; Rider, D. A.; Elias, A. L.; Thomas, M.; Harris, K. D.; Buriak, J. M. Advanced Functional Materials, 2011, 21, 1816-1826.

47. Mandal, U. K. Polymer International, 2002, 49, 1653-1657.

48. Cook, R. L.; Myers, A. W., Nanotechnology Applications in Coatings. 2009.

49. Liu, D. W., Wei; Qiu, Yongfu; Yang, Shihe; Xiao, Si; Wang, QuQuan; Ding, Lu; Wang, Jiannong. Langmuir, 2008, 24, 5052-5059.

50. Brown, P. J.; Thomas, D. S.; Köhler, A.; Wilson, J. S.; Kim, J.-S.; Ramsdale, C. M.; Sirringhaus, H.; Friend, R. H. Physical Review B, 2003, 67 (6), 064203.

51. Xue, B.; Vaughan, B.; Poh, C.-H.; Burke, K. B.; Thomsen, L.; Stapleton, A.; Zhou, X.; Bryant, G. W.; Belcher, W.; Dastoor, P. C. The Journal of Physical Chemistry C, 2010, 114 (37), 15797-15805.

52. Heo, S.; Park, K.; Seo, Y.; Ahmed, F.; Anwar, M. S.; Koo, B. Electron. Mater. Lett., 2013, 9 (3), 261-265.

53. Li, W.; Worfolk, B. J.; Li, P.; Hauger, T. C.; Harris, K. D.; Buriak, J. M. Journal of Materials Chemistry, 2012, 22, 11354-11363.

54. (a) Roy, S.; Aguirre, A.; Higgins, D. A.; Chikan, V. The Journal of Physical Chemistry C, 2011, 116 (4), 3153-3160; (b) Greenham, N. 
C.; Peng, X.; Alivisatos, A. P. Physical Review B, 1996, 54 (24), 17628-17637.

55. (a) Iwasaki, K.-i.; Fujimoto, H.; Matsuzaki, S. Synthetic Metals, 1994, 63 (2), 101-108; (b) Tashiro, K.; Ono, K.; Minagawa, Y.; Kobayashi, M.; Kawai, T.; Yoshino, K. Journal of Polymer Science Part B: Polymer Physics, 1991, 29 (10), 1223-1233. 